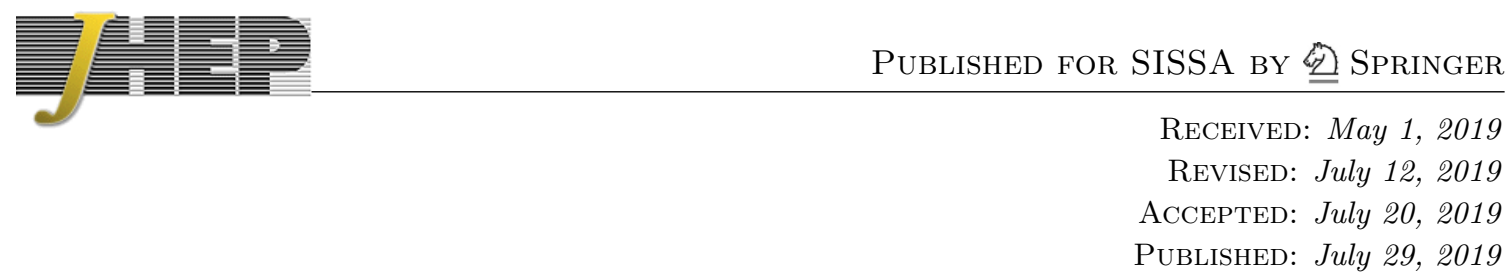

\title{
On scalaron decay via the trace of energy-momentum tensor
}

\author{
Ayuki Kamada \\ Center for Theoretical Physics of the Universe, \\ Institute for Basic Science (IBS), Daejeon 34126, Korea \\ E-mail: ayuki.kamada@gmail.com
}

ABSTRACT: In some inflation scenarios such as $R^{2}$ inflation, a gravitational scalar degrees of freedom called scalaron is identified as inflaton. Scalaron linearly couples to matter via the trace of energy-momentum tensor. We study scenarios with a sequestered matter sector, where the trace of energy-momentum tensor predominantly determines the scalaron coupling to matter. In a sequestered setup, heavy degrees of freedom are expected to decouple from low-energy dynamics. On the other hand, it is non-trivial to see the decoupling since scalaron couples to a mass term of heavy degrees of freedom. Actually, when heavy degrees of freedom carry some gauge charge, the amplitude of scalaron decay to two gauge bosons does not vanish in the heavy mass limit. Here a quantum contribution to the trace of energy-momentum tensor plays an essential role. This quantum contribution is known as trace anomaly or Weyl anomaly. The trace anomaly contribution from heavy degrees of freedom cancels with the contribution from the classical scalaron coupling to a mass term of heavy degrees of freedom. We see how trace anomaly appears both in the Fujikawa method and in dimensional renormalization. In dimensional renormalization, one can evaluate the scalaron decay amplitude in principle at all orders, while it is unclear how to process it beyond the one-loop level in the Fujikawa method. We consider scalaron decay to two gauge bosons via the trace of energy-momentum tensor in quantum electrodynamics with scalars and fermions. We evaluate the decay amplitude at the leading order to demonstrate the decoupling of heavy degrees of freedom.

Keywords: Anomalies in Field and String Theories, Cosmology of Theories beyond the SM

ArXiv EPRINT: 1902.05209

Open Access, (c) The Authors.

Article funded by $\mathrm{SCOAP}^{3}$.

https://doi.org/10.1007/JHEP07(2019)172 


\section{Contents}

1 Introduction 1

2 Gravitational coupling of scalaron to matter 3

3 Trace of energy-momentum tensor $\quad 6$

$\begin{array}{lll}4 & \text { Conclusion and remarks } & 10\end{array}$

$\begin{array}{ll}\text { A Gauge fixing term } & 10\end{array}$

B Path integral derivation of eq. (2.15) 11

C One-loop calculations in QED

$\begin{array}{lll}\text { C.1 Scalar } & 12\end{array}$

$\begin{array}{lll}\text { C.2 Fermion } & 15\end{array}$

$\begin{array}{lll}\text { C.3 Summary of one-loop functions } & 19\end{array}$

\section{Introduction}

Inflation is a cosmological paradigm that solves issues of big bang cosmology, such as the horizon, flatness, and monopole problems [1-8]. It also provides an almost scaleinvariant density contrast over homogeneous and isotropic background [9-15]. The inflation paradigm has been strongly supported by the deviation of the scalar spectral index from unity observed in cosmic microwave background anisotropies [16]. Among various inflation models [17], $R^{2}$ inflation ( $R$ : Ricci scalar) $[2,18-22]$ is a good benchmark. Its plateau potential predicts a tensor-to-scalar ratio sufficiently small to be consistent with the Planck data [16] but within a reach of future searches of cosmic microwave background $B$-mode anisotropies [23-25].

Identifying a reheating temperature $T_{R}$ in $R^{2}$ inflation is important for theoretical prediction of the scalar spectral index and tensor-to-scalar ratio [26]. It also plays an important role in production mechanisms of dark matter and baryon asymmetry [27, 28]. For example, $T_{R} \gtrsim 10^{9} \mathrm{GeV}$ (e.g., refs. [29-32]) is required for thermal leptogenesis [33] to work. Furthermore it is imprinted in the primordial gravitational wave spectrum when the energy density of Universe is transferred from oscillating inflaton to radiation [34]. Such an imprint could be seen in ultimate gravitational wave experiments [35].

In $f(R)$ gravity including $R^{2}$ inflation, a gravitational scalar degrees of freedom called scalaron is identified as inflaton. To determine the reheating temperature, we need to study scalaron coupling to matter. $f(R)$ gravity generically can be rewritten as a scalar-tensor theory through a Weyl transformation (local rescaling of the metric and fields) that is a function solely of scalaron $[19,36]$. This Weyl transformation manifests scalaron coupling to 
the trace of matter energy-momentum tensor in the scalaron frame [37]. ${ }^{1}$ Similar situations can also be seen in a broader class of inflation models based on a scalar-tensor theory. One example is $f(\sigma) R$ gravity (let us also refer to a scalar field $\sigma$ as scalaron) [38-42]. The trace of energy-momentum tensor predominantly determines scalaron coupling to matter, when scalaron direct coupling to matter in the Jordan frame is suppressed for some reason. In this paper, we consider such scenarios where a matter sector communicates with the scalaron sector only gravitationally in the Jordan frame.

Scalaron decay ${ }^{2}$ is dominated by decay channels to two scalars if their non-minimal coupling to Ricci curvature deviates from the conformal coupling. With the conformally coupled scalars, loop-induced decay to two gauge bosons becomes relevant. The decay amplitude is proportional to the $\beta$ function of the corresponding gauge coupling. Ref. [34] uses the $\beta$ function at the energy scale of the scalaron mass $\left(\simeq 3 \times 10^{13} \mathrm{GeV}\right.$ for the $R^{2}$ inflation model), which virtually counts light degrees of freedom. Refs. [43, 44], which study inflaton decay in $f(\sigma) R$ gravity, also virtually counts light degrees of freedom.

On the other hand, it is less manifest at first sight if heavy degrees of freedom do not contribute to scalaron decay. In the scalaron frame, scalaron couples to matter via mass terms. Loop-induced decay to two gauge bosons does not vanish in the heavy mass limit. It leaves scalaron coupling to gauge bosons for low-energy effective theory. ${ }^{3}$ Meanwhile the decoupling of heavy degrees of freedom may be apparent in the Jordan frame, where scalaron does not have any direct coupling to matter. Matter fields decouple in the heavy mass limit without leaving any non-decoupling effects for low-energy effective theory. This raises an issue on the "frame equivalence" (see also ref. [58] for a related discussion).

What plays an essential role is a quantum contribution to the trace of energymomentum tensor, known as Weyl anomaly or trace anomaly. ${ }^{4}$ Trace anomaly is intensively investigated both in the flat spacetime [59-70] and in a curved spacetime [7180] (see also ref. [81] for a review). The trace anomaly contribution from heavy degrees of freedom cancels with the contribution from the classical scalaron coupling to a mass term of heavy degrees of freedom. Because of the cancellation between classical and quantum contributions, the scalaron coupling to matter via the trace of energy-momentum tensor is ultraviolet insensitive. ${ }^{5}$

\footnotetext{
${ }^{1}$ Note that the Weyl transformation consists solely of scalaron. Therefore this is not the Einstein frame since scalar fields in a matter section (not scalaron) can still have a non-minimal coupling to the Ricci scalar.

${ }^{2}$ In this paper, we consider perturbative scalaron decay. We assume that non-perturbative effects associated with non-zero field values of scalaron and matter scalars are negligible. This could be true since decay proceeds only gravitationally and occurs long after inflation.

${ }^{3}$ This is the case for Higgs [45, 46] or axion [47-50] (see also refs. [51, 52] and [53, 54] for popular ultraviolet realizations). One famous example is coupling of Higgs [45, 46] or axion [47-50] (see also refs. $[51,52]$ and $[53,54]$ for popular ultraviolet realizations) to light gauge bosons such as photon or gluon in low-energy effective theory. With this observation, ref. [55] argues that one should count heavy degrees of freedom as well as light degrees of freedom for the $\beta$ function. This result is taken from ref. [56], which studies scalaron decay in $f(\sigma) R$ gravity. A similar calculation on scalaron coupling to the standard model particles has been made in ref. [57]. Their stance on the frame equivalence is different from the present study.

${ }^{4}$ The trace of energy-momentum tensor and trace anomaly are often not distinguished. In this paper we use the former to refer to the whole (classical + quantum) contribution, while we use the latter to refer to only a quantum contribution.

${ }^{5}$ This is analogous to an anomaly mediation contribution to a sparticle mass in supersymmetric theories [82, 83], which boasts its ultraviolet insensitivity. A quantum contribution to a gaugino mass from
} 
This paper is organized as follows. In the next section we describe scenarios with a sequestered matter sector, where scalaron couples to matter predominantly via the trace of energy-momentum tensor. We demonstrate how the trace of energy-momentum tensor receives a quantum contribution, by employing the Fujikawa method [87-89] (see also ref. [90] for a comprehensive summary). The Fujikawa method is illustrating trace anomaly, but not convenient in practical calculations such as perturbative renormalization. Instead, in section 3, we use dimensional renormalization, i.e., the minimal subtraction (MS) or modified minimal subtraction ( $\overline{\mathrm{MS}})$ scheme [91-93], where we can compute perturbative renormalization in principle at all orders. ${ }^{6}$ We see how trace anomaly appears in dimensional renormalization. Furthermore, we compute the leading amplitude of scalaron decay into two gauge boson in quantum electrodynamics (QED) with scalars and fermions. We see that heavy degrees of freedom do not contribute to the amplitude. section 4 is devoted to a summary and further remarks. We use a notation of ref. [94], where the four-dimension metric has the signature of $(+,-,-,-)$.

\section{Gravitational coupling of scalaron to matter}

We consider a class of inflation models where a scalaron sector communicates with a matter sector only gravitationally as

$$
S_{\text {grav }}\left[g_{\mu \nu}^{\prime}, \sigma^{\prime}\right]+S_{\text {mat }}\left[\left\{\phi_{i}^{\prime}\right\}, g_{\mu \nu}^{\prime} ;\left\{\lambda_{a}\right\}\right]
$$

in the Jordan frame. $g_{\mu \nu}$ is the metric and a prime denotes the quantity in the Jordan frame. $\left\{\phi_{i}\right\}$ and $\left\{\lambda_{a}\right\}$ collectively denote matter fields and parameters, respectively. Note that scalaron in $f(R)$ gravity is not manifest in the Jordan frame. For example, in the $R^{2}$ inflation model,

$$
S_{\text {grav }}=-\frac{M_{\mathrm{pl}}^{2}}{2} \int d^{4} x \sqrt{-g^{\prime}}\left(R^{\prime}-\frac{R^{\prime 2}}{6 \mu^{2}}\right),
$$

with the reduced Planck mass $M_{\mathrm{pl}} \simeq 2.435 \times 10^{18} \mathrm{GeV}$ and a mass parameter $\mu$.

We assume that the matter sector is minimally coupled to gravity, while maintaining renormalizability ${ }^{7}$ up to graviton loops that are suppressed by $1 / M_{\mathrm{pl}}^{2}$. In particular we require renormalizablity of energy-momentum tensor that is defined as a linear response of the matter action to the metric. For example, QED with a scalar $\phi$ is described by

$$
\begin{aligned}
S_{\mathrm{mat}}=\int d^{4} x \sqrt{-g^{\prime}}(- & \frac{1}{4} g^{\prime \mu \lambda} g^{\prime \nu \kappa} F_{\mu \nu}^{\prime} F_{\lambda \kappa}^{\prime}+g^{\prime \mu \nu} D_{\mu}^{\prime} \phi^{\prime *} D_{\nu}^{\prime} \phi^{\prime} \\
& \left.+\xi_{\mathrm{grav}} R^{\prime}\left|\phi^{\prime}\right|^{2}-m_{s}^{2}\left|\phi^{\prime}\right|^{2}-\frac{1}{4} \lambda\left|\phi^{\prime}\right|^{4}\right)+S_{\mathrm{fix}}
\end{aligned}
$$

heavy degrees of freedom cancels with a classical contribution from a coupling of a compensator field to a mass term of heavy degrees of freedom. Indeed superconformal anomaly is correctly taken into account in supersymmetric inflation setups [84-86].

${ }^{6}$ Ref. [44] sketches the derivation of trace anomaly at the one-loop order in Wilsonian renormalization.

${ }^{7}$ This does not mean the matter sector consists solely of a finite number of renormalizable terms. Nonrenormalizable terms are allowed when an infinite number of non-renormalizable terms are introduced for renormalization in the usual sense of effective field theory. 
with $D_{\mu}$ being the gauge and diffeomorphism covariant derivative and $F_{\mu \nu}$ being the field strength of $A_{\mu} \cdot m_{s}$ is a scalar mass and $\lambda$ is a quartic coupling. A non-minimal coupling $\xi_{\text {grav }}$, which provides an improvement term of energy-momentum tensor [59,60], should be kept to maintain renormalizability of energy-momentum tensor. We devote section A to the gauge fixing term $S_{\text {fix }}$, whose contribution to energy-momentum tensor can be omitted for physical states.

Via the Weyl transformation of

$$
g_{\mu \nu}^{\prime}=e^{2 \omega(\sigma)} g_{\mu \nu}
$$

the scalaron + gravity sector turns into $S_{\text {grav }}=S_{\mathrm{E}-\mathrm{H}}+S_{\sigma}$ :

$$
\begin{aligned}
S_{\mathrm{E}-\mathrm{H}} & =-\frac{M_{\mathrm{pl}}^{2}}{2} \int d^{4} x \sqrt{-g} R, \\
S_{\sigma} & =\int d^{4} x \sqrt{-g}\left(\frac{1}{2} g^{\mu \nu} \nabla_{\mu} \sigma \nabla_{\nu} \sigma-V(\sigma)\right),
\end{aligned}
$$

with $\nabla_{\mu}$ being the diffeomorphism covariant derivative. For example, in the $R^{2}$ inflation model,

$$
\omega=-\frac{1}{\sqrt{6}} \frac{\sigma}{M_{\mathrm{pl}}}
$$

and

$$
V(\sigma)=\frac{3}{4} \mu^{2} M_{\mathrm{pl}}^{2}\left[1-\exp \left(-\sqrt{\frac{2}{3}} \frac{\sigma}{M_{\mathrm{pl}}}\right)\right]^{2} .
$$

The matter fields transform under the Weyl transformation as

$$
\phi_{i}^{\prime}=e^{-d_{i} \omega(\sigma)} \phi_{i}
$$

with $d_{i}$ denoting the Weyl weight of the field $\phi_{i}$. The linear variation of the matter action is responsible for the leading coupling of scalaron to matter:

$$
S_{\mathrm{mat}}\left[\left\{\phi_{i}^{\prime}\right\}, g_{\mu \nu}^{\prime} ;\left\{\lambda_{a}\right\}\right] \simeq S_{\mathrm{mat}}\left[\left\{\phi_{i}\right\}, g_{\mu \nu} ;\left\{\lambda_{a}\right\}\right]-\int d^{4} x \sqrt{-g} \omega(\sigma) A_{\operatorname{lin}}\left(\left\{\phi_{i}\right\}, g_{\mu \nu} ;\left\{\lambda_{a}\right\}\right)
$$

When we treat fields as classical objects, it is given by

$$
A_{\text {lin }}^{\text {class }}=-\sum_{i} d_{i}(\text { e.o.m. })_{i}+\left(g_{\mu \nu} T^{\mu \nu}\left(\left\{\phi_{i}\right\}, g_{\mu \nu} ;\left\{\lambda_{a}\right\}\right)\right)_{\text {class }}
$$

and

$$
\text { (e.o.m. })_{i}=-\phi_{i} \frac{1}{\sqrt{-g}} \frac{\delta S_{\mathrm{mat}}\left[\left\{\phi_{i}\right\}, g_{\mu \nu} ;\left\{\lambda_{a}\right\}\right]}{\delta \phi_{i}}
$$


The second term of $A_{\text {lin }}^{\text {class }}$ is the classical trace of energy-momentum tensor, in which we treat fields as classical objects. We define energy-momentum tensor by a functional derivative of

$$
T^{\mu \nu}=-\frac{2}{\sqrt{-g}} \frac{\delta S_{\mathrm{mat}}\left[\left\{\phi_{i}\right\}, g_{\mu \nu} ;\left\{\lambda_{a}\right\}\right]}{\delta g_{\mu \nu}}
$$

For example, in scalar QED,

$$
\begin{aligned}
T_{\mu \nu}= & -g^{\lambda \kappa} F_{\mu \lambda} F_{\nu \kappa}+2 D_{\mu} \phi^{*} D_{\nu} \phi+2 \xi_{\mathrm{grav}} R_{\mu \nu}|\phi|^{2}-2 \xi_{\mathrm{grav}}\left(\nabla_{\mu} \nabla_{\nu}-g_{\mu \nu} g^{\lambda \kappa} \nabla_{\lambda} \nabla_{\kappa}\right)|\phi|^{2} \\
& -g_{\mu \nu}\left(-\frac{1}{4} g^{\lambda \rho} g^{\kappa \sigma} F_{\lambda \kappa} F_{\rho \sigma}+g^{\lambda \kappa} D_{\lambda} \phi^{*} D_{\kappa} \phi+\xi_{\mathrm{grav}} R|\phi|^{2}-m^{2}|\phi|^{2}-\frac{1}{4} \lambda|\phi|^{4}\right) .
\end{aligned}
$$

When we treat fields as quantum operators, the linear variation $A_{\text {lin }}$ receives an additional contribution $A_{\text {anom }}$. To see it, let us take a path integral formalism with path integral measure of $\mathcal{D}\left\{\phi_{i}^{\prime}\right\}\left[g_{\mu \nu}^{\prime}\right]$. Note that the path integral measure depends on the metric such that the path integral is diffeomorphism invariant [90]. For example, for scalar QED, $\mathcal{D} \phi\left[g_{\mu \nu}\right]=\mathcal{D}(-g)^{1 / 4} \phi$ and $\mathcal{D} A_{\mu}\left[g_{\mu \nu}\right]=\mathcal{D}(-g)^{1 / 4} e_{m}^{\mu} A_{\mu}$, where $e_{\mu}^{m}$ is the vierbein. We change the variables from $\left\{\phi_{i}^{\prime}\right\}$ in the left hand side to $\left\{\phi_{i}\right\}$ in the right hand side of eq. (2.9). This results in a Jacobian of path integral measure:

$$
\mathcal{D}\left\{\phi_{i}^{\prime}\right\}\left[g_{\mu \nu}^{\prime}\right] \simeq \mathcal{D}\left\{\phi_{i}\right\}\left[g_{\mu \nu}\right] \exp \left(-i \int d^{4} x \sqrt{-g} \omega A_{\text {Jacob }}\left(\left\{\phi_{i}\right\}, g_{\mu \nu} ;\left\{\lambda_{a}\right\}\right)\right)
$$

in the linear variation. One may evaluate $A_{\text {Jacob }}$ by using heat kernel regularization, which is used in Fujikawa's derivation of chiral anomaly [95]. It provides a one-loop contribution to $A_{\text {anom }}$, which is proportional to the Weyl tensor squared, the Gauss-Bonnet density, and a gauge field strength squared if $\left\{\phi_{i}\right\}$ is charged. One can identify $A_{\text {Jacob }}=A_{\text {anom }}$, which is Fujikawa's derivation of trace anomaly [90]. It follows that the linear variation $A_{\text {lin }}$ is given by the quantum trace of energy-momentum tensor (see section $\mathrm{B}$ ):

$$
\begin{aligned}
A_{\operatorname{lin}} & =-\sum_{i} d_{i}(\text { e.o.m. })_{i}+\left(g_{\mu \nu} T^{\mu \nu}\right)_{\text {class }}+A_{\text {anom }}\left(\left\{\phi_{i}\right\}, g_{\mu \nu} ;\left\{\lambda_{a}\right\}\right) \\
& =-\sum_{i} d_{i}(\text { e.o.m. })_{i}+g_{\mu \nu} T^{\mu \nu}\left(\left\{\phi_{i}\right\}, g_{\mu \nu} ;\left\{\lambda_{a}\right\}\right) .
\end{aligned}
$$

In the above discussion, we have taken into account a Jacobian of path integral measure associated with $\left\{\phi_{i}^{\prime}\right\} \rightarrow\left\{\phi_{i}\right\}$ under a background metric. One also needs to care a Jacobian of path integral measure associated with $g_{\mu \nu}^{\prime} \rightarrow g_{\mu \nu}$ in eq. (2.4). On the other hand, it is intricate to compute the gravitational Jacobian. Thus we just assume that it does not give rise to any relevant coupling between scalaron and matter. For example, in the $R^{2}$ inflation model, the scalaron coupling to matter in eq. (2.9) reads

$$
S_{\sigma-\mathrm{mat}}=\int d^{4} x \sqrt{-g} \frac{1}{\sqrt{6}} \frac{\sigma}{M_{\mathrm{pl}}} g_{\mu \nu} T^{\mu \nu} .
$$


Our assumption on the gravitational Jacobian reads that it only leads to couplings suppressed by a higher power of $1 / M_{\mathrm{pl}}$. This could be true since the graviton-loop contribution is suppressed by $1 / M_{\mathrm{pl}}^{2}$.

In the rest of this paper, we restrict our discussion within the flat spacetime. The trace of flat-spacetime energy-momentum tensor is enough to evaluate scalaron decay since the scalaron decay amplitude into graviton is further suppressed by $1 / M_{\mathrm{pl}}$.

\section{Trace of energy-momentum tensor}

In the last section we have shown that in the scalaron frame the scalaron couples to matter via the quantum trace of energy-momentum tensor, by employing the Fujikawa method. Here we should remark that once we use some regularization, we need to use it throughout, for example, to calculate the renormalization of couplings $\left\{\lambda_{a}\right\}$. On the other hand, heat kernel regularization in the Fujikawa method is not practical for perturbative renormalization, for which dimensional renormalization is a usual choice. ${ }^{8}$ In dimensional renormalization, we consider $d=4-\epsilon$ dimension instead of four dimension to make loop diagrams finite. Then we subtract divergences in the four-dimension limit such that counter terms compose solely of poles of $\epsilon$.

In dimensional renormalization, $A_{\text {Jacob }}$ does not depend on fields unlike that in the Fujikawa method with heat kernel regularization. Thus $A_{\text {anom }}$ has a different origin in dimensional renormalization. The trace of energy-momentum tensor takes a form of

$$
T_{\mu}^{\mu}=\lim _{\epsilon \rightarrow 0}\left(-\sum_{i} d_{i}(\text { e.o.m. })_{i}+\left(T_{\mu}^{\mu}\right)_{\text {class }}\right) .
$$

In the right-hand side, a quantity inside the parenthesis is calculated in $d=4-\epsilon$ dimension and then taken to the four-dimension limit of $\epsilon \rightarrow 0$. A key observation is that as $\epsilon \rightarrow 0$, the second term does not coincide with the four-dimension classical trace of energy-momentum tensor. This is because of renormalization (normal product) of the bare (composite) operators such as $F_{\mu \nu}^{2}$ and $|\phi|^{4}[102-105]$. The renormalization coefficients, including the multiplicative renormalization of bare couplings such as $\lambda$, compose of subtracted poles of $\epsilon$ in the MS or $\overline{\mathrm{MS}}$ scheme. They lead to terms proportional to the $\beta$ function of the renormalized couplings $[65,67]$ such as $\beta_{e}\left[\bar{F}_{\mu \nu}^{2}\right]$ and $\beta_{\lambda}\left[|\bar{\phi}|^{4}\right]$. A bar denotes the renormalized (not composite) fields and parameters and a square bracket denotes the renormalized composite operator. These contributions provide $A_{\text {anom }}$. Also note that $T_{\mu \nu}$ is conserved and thus solely improvement terms arising from non-minimal couplings are renormalized. As a result $T_{\mu}^{\mu}$ is already finite up to renormalization of improvement terms. In this article we do not go into further detail about renormalization of improvement terms, since it does not change the result at the leading order.

\footnotetext{
${ }^{8}$ Here is a big difference between chiral anomaly and trace anomaly. Chiral anomaly takes a one-loop exact form [96, 97] up to the divergence of some gauge invariant current [98] due to its topological property, i.e., it counts a number of zero modes in an instanton background [99-101]. Thus one can use the result from heat kernel regularization even though one uses dimensional regularization for perturbative renormalization. On the other hand, it does not hold for trace anomaly.
} 
For scalar QED, the Lagrangian density is given by

$$
\mathcal{L}=-\frac{1}{4} F_{\mu \nu}^{2}-\frac{1}{2 \xi}\left(\partial_{\mu} A^{\mu}\right)^{2}+\left|D_{\mu} \phi\right|^{2}-m_{s}^{2}|\phi|^{2}-\frac{1}{4} \lambda|\phi|^{4},
$$

with $D_{\mu}=\partial_{\mu}-i q e A_{\mu}$ being the gauge covariant derivative for a charge $q$. We have integrated out the Nakanishi-Lautrup [106, 107] and (anti-)ghost fields (see section A). $\xi$ is a gauge fixing parameter. ${ }^{9} d$-dimension flat-spacetime energy-momentum tensor is obtained from eq. (2.13) as

$$
\begin{aligned}
T_{\mu \nu}= & -g^{\lambda \kappa} F_{\mu \lambda} F_{\nu \kappa}+2 D_{\mu} \phi^{*} D_{\nu} \phi-2\left(\xi_{\text {grav }}^{c}+\frac{\eta}{d-1}\right)\left(\partial_{\mu} \partial_{\nu}-g_{\mu \nu} \partial^{2}\right)|\phi|^{2} \\
& -g_{\mu \nu}\left(-\frac{1}{4} F_{\lambda \kappa}^{2}+\left|D_{\mu} \phi\right|^{2}-m_{s}^{2}|\phi|^{2}-\frac{1}{4} \lambda|\phi|^{4}\right) .
\end{aligned}
$$

where we rewrite $\xi_{\text {grav }}=\xi_{\text {grav }}^{c}+\eta /(d-1)$ with $\xi_{\text {grav }}^{c}=(d-2) /(4(d-1))$ in $d$ dimension. We remark that $\eta$ is renormalized in a non-multiplicative manner to make $T_{\mu \nu}$ finite, although we do not go into further detail. Taking a classical trace, one finds

$$
\left(T_{\mu}^{\mu}\right)_{\text {class }}=\epsilon\left(-\frac{1}{4} F_{\mu \nu}^{2}+\frac{1}{4} \lambda|\phi|^{4}\right)+2 m_{s}^{2}|\phi|^{2}+2 \eta \partial^{2}|\phi|^{2}+\left(1-\frac{\epsilon}{2}\right)(\text { e.o.m })
$$

where the last term with

$$
\text { (e.o.m })=\phi^{*}\left(D^{2} \phi+m_{s}^{2} \phi+\frac{2}{4} \lambda|\phi|^{2} \phi\right)+\left(D^{2} \phi^{*}+m_{s}^{2} \phi^{*}+\frac{2}{4} \lambda|\phi|^{2} \phi^{*}\right) \phi
$$

cancels with $-\sum_{i} d_{i}$ (e.o.m. $)_{i}$ in eq. (2.15). The first term of $\left(T_{\mu}^{\mu}\right)_{\text {class }}$ vanishes at the classical level as $\epsilon \rightarrow 0$, but not at the quantum level. This contribution provides $A_{\text {anom }}$.

We calculate a $T_{\mu}^{\mu}{ }^{-} \bar{A}_{\lambda}-\bar{A}_{\kappa}\left(\bar{A}_{\mu}\right.$ : renormalized gauge field) correlation function in the scalaron frame by using the $\overline{\mathrm{MS}}$ scheme. More specifically, we calculate the amputated amplitude $\mathcal{M}_{T A A}$ with incoming momentum $k$ through $T_{\mu}^{\mu}$ and outgoing momentum $k_{1}$ and $k_{2}$ through gauge bosons with helicity $\epsilon_{1}$ and $\epsilon_{2}$, respectively. section $\mathrm{C}$ is devoted to details of the computations. For example, in the $R^{2}$ inflation model, the invariant amplitude of scalaron decay into two gauge bosons is given by

$$
\mathcal{M}_{\mathrm{dec}}=\frac{1}{\sqrt{6}} \frac{1}{M_{\mathrm{pl}}} \mathcal{M}_{T A A} \times Z_{3}^{\text {pole }} .
$$

Here, the last term $Z_{3}^{\text {pole }}$, which is the residue of the mass pole of the gauge field, arises from the Lehmann-Symanzik-Zimmermann (LSZ) reduction formula [108].

For scalar QED (see section C.1), the leading contribution to $\mathcal{M}_{T A A}$ arises from the following terms of the trace of energy-momentum tensor: ${ }^{10}$

$$
T_{\mu}^{\mu} \supset \frac{1}{6} \frac{q^{2} \bar{e}^{2}}{16 \pi^{2}} \bar{F}_{\mu \nu}^{2}+2 \bar{m}^{2}|\bar{\phi}|^{2}+2 \bar{\eta} \partial^{2}|\bar{\phi}|^{2}
$$

\footnotetext{
${ }^{9}$ Note that a gauge fixing parameter $\xi$ is different from a non-minimal coupling $\xi_{\text {grav }}$.

${ }^{10}$ Note that in general $\bar{F}_{\mu \nu}^{2} \neq\left[F_{\mu \nu}^{2}\right]$, although they coincide with each other at this order.
} 

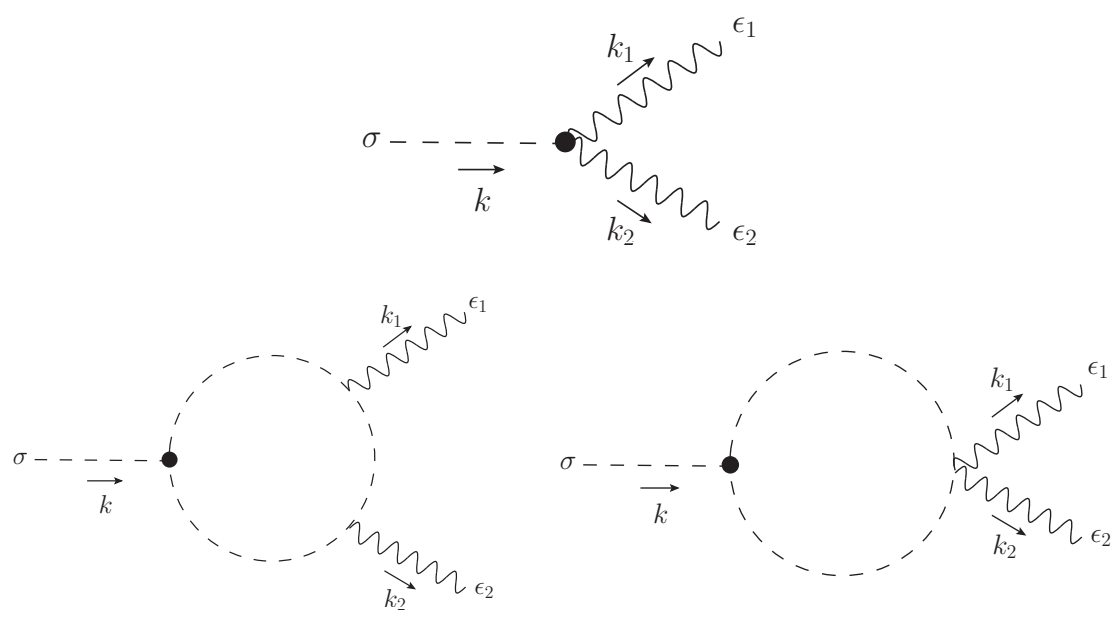

Figure 1. The leading contributions to $\mathcal{M}_{T A A}$, i.e., $T_{\mu}^{\mu}-\bar{A}_{\lambda}-\bar{A}_{\kappa}$ correlation function ( $\sigma$ decay into two gauge bosons). [Top] $\mathcal{M}_{F^{2}}$ : the gauge kinetic term in eq. (3.7) is inserted. [Bottom] $\mathcal{M}_{|\phi|^{2}}$ : the scalar mass term and $\eta$ term in eq. (3.7) is inserted. There is the other contribution from the left diagram with the external gauge bosons exchanged.

The first term arises from the gauge kinetic term proportional to $\epsilon$ in eq. (3.4). Its coefficient is obtained from the leading contribution to the wave function renormalization of the gauge field [see eq. (C.9)]. Meanwhile the leading contribution to the wave function renormalization of the gauge field also determines the leading contribution to the $\beta$ function [see eq. (C.10)] as

$$
\beta_{e}=\frac{1}{3} \frac{q^{2} \bar{e}^{3}}{16 \pi^{2}}
$$

The matrix element has two contributions*

$$
\mathcal{M}_{T A A}=\mathcal{M}_{F^{2}}+\mathcal{M}_{|\phi|^{2}} .
$$

The first term arises from the tree-level diagram with the gauge kinetic term inserted (see the top diagram of figure 1$)$ :

$$
\mathcal{M}_{F^{2}}=-\frac{2}{3} \frac{q^{2} \bar{e}^{2}}{16 \pi^{2}}\left(k_{1} \cdot k_{2} \epsilon_{1}^{*} \cdot \epsilon_{2}^{*}-k_{2} \cdot \epsilon_{1}^{*} k_{1} \cdot \epsilon_{2}^{*}\right) .
$$

The second term arises from the one-loop diagram with the scalar mass term and $\eta$ term inserted (see the bottom diagrams of figure 1):

$$
\mathcal{M}_{|\phi|^{2}}=\frac{2}{3} \frac{q^{2} \bar{e}^{2}}{16 \pi^{2}} \frac{\bar{m}^{2}-\bar{\eta} k^{2}}{\bar{m}^{2}} I_{s}\left(\frac{k^{2}}{\bar{m}^{2}}\right)\left(k_{1} \cdot k_{2} \epsilon_{1}^{*} \cdot \epsilon_{2}^{*}-k_{2} \cdot \epsilon_{1}^{*} k_{1} \cdot \epsilon_{2}^{*}\right)
$$

where $^{11}$

$$
\begin{aligned}
I_{s}(r)= & 24 \int_{0}^{1} d x \int_{0}^{1-x} d y \frac{x y}{-r x y+1-i \epsilon_{\mathrm{ad}}} \\
& = \begin{cases}\frac{12}{r}\left(-1+\frac{4}{r} \arcsin ^{2} \frac{\sqrt{r}}{2}\right) & (\text { for } r<4) \\
\frac{12}{r}\left(-1-\frac{4}{r}\left[\operatorname{arccosh} \frac{\sqrt{r}}{2}-i \frac{\pi}{2}\right]^{2}\right) & (\text { for } r>4)\end{cases}
\end{aligned}
$$

\footnotetext{
${ }^{11}$ This definition is different from the one in ref. [56] by a factor of 6 .
} 
For $r>4$, one needs to take into account an adiabatic parameter $\epsilon_{\mathrm{ad}}>0$ properly. ${ }^{12}$ This arises from the fact that the loop scalar can be real. Collecting the two contributions, one obtains

$$
\mathcal{M}_{T A A}=-\frac{2}{3} \frac{q^{2} \bar{e}^{2}}{16 \pi^{2}}\left(1-\frac{\bar{m}^{2}-\bar{\eta} k^{2}}{\bar{m}^{2}} I_{s}\left(\frac{k^{2}}{\bar{m}^{2}}\right)\right)\left(k_{1} \cdot k_{2} \epsilon_{1}^{*} \cdot \epsilon_{2}^{*}-k_{2} \cdot \epsilon_{1}^{*} k_{1} \cdot \epsilon_{2}^{*}\right) .
$$

We remark that $I_{s}(0)=1$ and thus a heavy $\left(\bar{m}^{2} \gg k^{2}\right)$ scalar does not contribute to $\mathcal{M}_{T A A}$. Meanwhile, $I_{s}(r \rightarrow \infty) \rightarrow-12 / r$ and thus a light $\left(\bar{m}^{2} \ll k^{2}\right)$ scalar indeed contributes to $\mathcal{M}_{T A A}$.

It is straightforward to generalize to the case with $N_{s}$ scalars and $N_{f}$ Dirac fermions (see section C.2 for QED with a Dirac fermion) since the quartic and Yukawa coupling do not matter at this order. The $\beta$ function is given by

$$
\beta_{e}=\frac{1}{3}\left(\sum_{s} q_{s}^{2}+4 \sum_{f} q_{f}^{2}\right) \frac{\bar{e}^{3}}{16 \pi^{2}}
$$

Note that this counts contributions from both heavy and light degrees of freedom.

Meanwhile, the matrix element is given by

$$
\begin{aligned}
\mathcal{M}_{T A A}= & \frac{2}{3} \frac{\bar{e}^{2}}{16 \pi^{2}}\left(\sum_{s} q_{s}^{2}\left(1-\frac{\bar{m}_{s}^{2}-\bar{\eta}_{s} k^{2}}{\bar{m}_{s}^{2}} I_{s}\left(\frac{k^{2}}{\bar{m}_{s}^{2}}\right)\right)+4 \sum_{f} q_{f}^{2}\left(1-I_{f}\left(\frac{k^{2}}{\bar{m}_{f}^{2}}\right)\right)\right) \\
& \times\left(k_{1} \cdot k_{2} \epsilon_{1}^{*} \cdot \epsilon_{2}^{*}-k_{2} \cdot \epsilon_{1}^{*} k_{1} \cdot \epsilon_{2}^{*}\right),
\end{aligned}
$$

where

$$
\begin{aligned}
I_{f}(r) & =3 \int_{0}^{1} d x \int_{0}^{1-x} d y \frac{-4 x y+1}{-r x y+1-i \epsilon_{\mathrm{ad}}} \\
& = \begin{cases}\frac{6}{r}\left(1+\left(1-\frac{4}{r}\right) \arcsin ^{2} \frac{\sqrt{r}}{2}\right) & (\text { for } r<4) \\
\frac{6}{r}\left(1-\left(1-\frac{4}{r}\right)\left[\operatorname{arccosh} \frac{\sqrt{r}}{2}-i \frac{\pi}{2}\right]^{2}\right) & (\text { for } r>4)\end{cases}
\end{aligned}
$$

Here $I_{f}(0)=1$ and $I_{f}(\infty)=0 .{ }^{13}$ For $r>4$, one needs to take into account $\epsilon_{\text {ad }}$ properly. This arises from the fact that the loop fermion can be real. The matrix element is approximated by

$$
\mathcal{M}_{T A A} \approx \frac{2}{3} \frac{\bar{e}^{2}}{16 \pi^{2}}\left(\sum_{\text {light } s} q_{s}^{2}\left(1-12 \bar{\eta}_{s}\right)+4 \sum_{\text {light } f} q_{f}^{2}\right)\left(k_{1} \cdot k_{2} \epsilon_{1}^{*} \cdot \epsilon_{2}^{*}-k_{2} \cdot \epsilon_{1}^{*} k_{1} \cdot \epsilon_{2}^{*}\right) .
$$

The summation runs over solely light scalars or fermions with $m^{2}<k^{2}$. The light scalar contribution is not only from the $\beta$ function $q_{s}^{2}$, but also from the non-minimal coupling $\bar{\eta}$. This is because the classical contribution to $T_{\mu}^{\mu}$ from the non-minimal coupling (the last term in eq. (3.7)) does not vanish for a light scalar.

\footnotetext{
${ }^{12}$ Note that an adiabatic parameter $\epsilon_{\text {ad }}$ associated with a Wick rotation is different from $\epsilon=4-d$ for dimensional regularization.

${ }^{13}$ This definition is different from the one in ref. [56] by a factor of 3.
} 


\section{Conclusion and remarks}

In this article, we have revisited scalaron decay via the trace of energy-momentum tensor. In particular we have studied scenarios with a sequestered matter sector, where the trace of energy-momentum tensor gives a dominant contribution to scalaron-matter coupling. We have referred to the $R^{2}$ inflation mode to be concrete. On the other hand, one can straightforwardly apply our results and discussions to more general $f(R)$ and $f(\sigma) R$ gravity models. One just modifies the relation between the Weyl parameter and scalaron accordingly. We have shown how trace anomaly arises by employing the Fujikawa method and dimensional renormalization. For perturbative renormalization beyond the one-loop level, the dimensional renormalization is more convenient than the Fujikawa method.

Trace anomaly plays an important role in ensuring that the trace of energy-momentum tensor is predictive in terms of low-energy effective theory. We have explicitly calculated the scalaron decay amplitude at the leading order in QED with scalars and fermions. The contribution of heavy degrees of freedom from trace anomaly cancels with the one from the mass term, in the heavy mass limit of the scalars and fermions. It is straightforward to generalize the discussion to quantum chromodynamics (QCD).

There are two caveats on the predictability of the trace of energy-momentum tensor: a non-minimal coupling of matter scalars to Ricci curvature; and the renormalizationscale dependence. They only appear in energy-momentum tensor and thus one cannot be determined its renormalized value through usual experiments unless graviton is involved in a process. Since a non-minimal coupling is required to renormalize energy-momentum tensor, one should keep it even when one considers a matter sector minimally coupled to gravity. In addition, it may not be clear how we can see that the scalaron decay amplitude is independent of the renormalization scale, since the trace of energy-momentum tensor is a composite operator. We will give a detailed discussion on these caveats somewhere else.

\section{Acknowledgments}

The work of A. K. is supported by IBS under the project code, IBS-R018-D1. A. K. gratefully thanks Heejung Kim, Takumi Kuwahara, and Kazuya Yonekura for valuable discussions. A. K. thanks Taishi Katsuragawa, Shinya Matsuzaki and Yuki Watanabe for discussions on refs. [55-57]. A. K. would also like to thank Ryusuke Jinno and Kohei Kamada for encouraging A. K. to work on this paper and providing comments on the manuscript.

\section{A Gauge fixing term}

In this section we discuss the gauge fixing term $S_{\text {fix }}$ in non-Abelian gauge theory, while we consider Abelian gauge theory (QED) in the main text. The gauge fixing term takes a Becchi-Rouet-Stora-Tyutin (BRST) form [109-113] of

$$
S_{\mathrm{fix}}=\int d^{d} x \sqrt{-g}\left(\frac{\xi}{2} B^{a} B^{a}-g^{\mu \nu} \nabla_{\mu} B^{a} A^{a}{ }_{\nu}+g^{\mu \nu} \nabla_{\mu} \bar{c}^{a} D_{\nu} c^{a}\right)
$$


with $\xi$ being a gauge fixing parameter. The superscript $a$ runs over gauge group generators $T^{a}\left[T^{a}=\mathbb{I}\right.$ (identity matrix) in QED]. $D_{\mu}$ is the gauge and diffeomorphism covariant derivative, while $\nabla_{\mu}$ is the diffeomorphism (not gauge) covariant derivative. We have introduced a bosonic auxiliary Nakanishi-Lautrup field $B^{a}=B^{a \dagger}$, fermionic (ghost and anti-ghost) fields, $c^{a}=c^{a \dagger}$ and $\bar{c}^{a}=-\bar{c}^{a \dagger}$.

The BRST transformation is defined by the following fermionic global transformation:

$$
\begin{aligned}
Q A_{\mu} & =D_{\mu} c, \\
Q c & =\frac{i}{2} e[c, c], \\
Q \bar{c} & =B, \\
Q B & =0,
\end{aligned}
$$

with $e$ being a gauge coupling. We have used the matrix notation of $A_{\mu}=A_{\mu}^{a} T^{a}$ and $D_{\mu} c=\partial_{\mu} c-i e[c, A]$, and so on. These are understood as $\left[Q, A_{\mu}\right]=i D_{\mu} c$ (commutator), $\{Q, \bar{c}\}=i B$ (anti-commutator), and so on in the operator formalism with $Q^{\dagger}=Q$. An operator or state is called BRST closed when it vanishes under the BRST transformation. Gauge invariant operators, such as a gauge invariant part of an action and its contribution to energy-momentum tensor [see eq. (2.13)], are BRST closed. Meanwhile an operator or state is called BRST exact when it can be written as the BRST transformation of some operator or state. Notably the gauge fixing term is BRST exact:

$$
S_{\mathrm{fix}}=\int d^{d} x \sqrt{-g} Q\left(\frac{\xi}{2} \bar{c}^{a} B^{a}-g^{\mu \nu} \nabla_{\mu} \bar{c}^{a} A_{\nu}^{a}\right) .
$$

$S_{\text {fix }}$ contribution to energy-momentum tensor is also BRST exact:

$$
T_{\mu \nu}^{\mathrm{fix}}=Q\left(-\nabla_{\mu} \bar{c}^{a} A^{a}{ }_{\nu}-\nabla_{\nu} \bar{c}^{a} A^{a}{ }_{\mu}-g_{\mu \nu}\left(\frac{\xi}{2} \bar{c}^{a} B^{a}-g^{\lambda \kappa} \nabla_{\lambda} \bar{c}^{a} A^{a}{ }_{\kappa}\right)\right) .
$$

One can see that the BRST transformation is nilpotent: $Q^{2}=0$. Thus a BRSTexact operator or state is BRST closed. We can introduce an equivalence class on the set of BRST-closed operators or states $\mathcal{H}_{\text {closed }}$ as $\mathcal{H}_{\text {closed }} \sim \mathcal{H}_{\text {closed }}+\mathcal{H}_{\text {exact }}$ with the set of BRST-exact operators or states $\mathcal{H}_{\text {exact }} \subset \mathcal{H}_{\text {closed }}$. The physical operator or state is defined by the quotient set of $\mathcal{H}_{\text {closed }} / \mathcal{H}_{\text {exact }}[114-116]$. Since $T_{\mu \nu}^{\text {fix }}$ is BRST exact, one can chose a physical representative such that $T_{\mu \nu}^{\mathrm{fix}}=0$.

\section{B Path integral derivation of eq. (2.15)}

We consider a correlation function in the path integral formalism:

$$
\begin{aligned}
\int \mathcal{D}\left\{\phi_{i}\right\}\left[g_{\mu \nu}^{\prime}\right] \exp & \left(i S_{\mathrm{mat}}\left[\left\{\phi_{i}\right\}, g_{\mu \nu}^{\prime} ;\left\{\lambda_{a}\right\}\right]\right) \prod\left\{\phi_{i}\right\} \\
\simeq & \left(1+\int d^{4} x \sqrt{-g} \omega g_{\mu \nu} \frac{2}{\sqrt{-g}} \frac{\delta}{\delta g_{\mu \nu}}\right) \\
& \times \int \mathcal{D}\left\{\phi_{i}\right\}\left[g_{\mu \nu}\right] \exp \left(i S_{\mathrm{mat}}\left[\left\{\phi_{i}\right\}, g_{\mu \nu} ;\left\{\lambda_{a}\right\}\right]\right) \prod\left\{\phi_{i}\right\}
\end{aligned}
$$


Meanwhile,

$$
\begin{aligned}
\int \mathcal{D} & \left\{\phi_{i}\right\}\left[g_{\mu \nu}^{\prime}\right] \exp \left(i S_{\text {mat }}\left[\left\{\phi_{i}\right\}, g_{\mu \nu}^{\prime} ;\left\{\lambda_{a}\right\}\right]\right) \prod\left\{\phi_{i}\right\} \\
= & \int \mathcal{D}\left\{\phi_{i}^{\prime}\right\}\left[g_{\mu \nu}^{\prime}\right] \exp \left(i S_{\text {mat }}\left[\left\{\phi_{i}^{\prime}\right\}, g_{\mu \nu}^{\prime} ;\left\{\lambda_{a}\right\}\right]\right) \prod\left\{\phi_{i}^{\prime}\right\} \\
\simeq & \int \mathcal{D}\left\{\phi_{i}\right\}\left[g_{\mu \nu}\right] \exp \left(i S_{\text {mat }}\left[\left\{\phi_{i}\right\}, g_{\mu \nu} ;\left\{\lambda_{a}\right\}\right]\right) \\
& \times\left(1+i \int d^{4} x \sqrt{-g} \omega\left[\sum_{i} d_{i}(\text { e.o.m. })_{i}-g_{\mu \nu} T^{\mu \nu}\left(\left\{\phi_{i}\right\}, g_{\mu \nu} ;\left\{\lambda_{a}\right\}\right)\right.\right. \\
& \left.\left.-A_{\text {Jacob }}\left(\left\{\phi_{i}\right\}, g_{\mu \nu} ;\left\{\lambda_{a}\right\}\right)\right]-\int d^{4} x \sqrt{-g} \omega \sum_{i} \frac{d_{i} \phi_{i}}{\sqrt{-g}} \frac{\delta}{\delta \phi_{i}}\right) \prod\left\{\phi_{i}\right\}
\end{aligned}
$$

In the first equality, we change a notation of the integration variable $\left\{\phi_{i}\right\}$, which has no physical effect. From this Ward-Takahashi identity, one finds

$$
-g_{\mu \nu} T^{\mu \nu}=-\left(g_{\mu \nu} T^{\mu \nu}\right)_{\text {class }}-A_{\mathrm{Jacob}}\left(\left\{\phi_{i}\right\}, g_{\mu \nu} ;\left\{\lambda_{a}\right\}\right)
$$

by ignoring the contact terms.

\section{One-loop calculations in QED}

In the following calculations, we use the $\overline{\mathrm{MS}}$ scheme with a spacetime dimension of $d=4-\epsilon$ and a renormalization scale of $\mu$, while compensating a mass dimension by a modified renormalization scale $\tilde{\mu}$ defined by

$$
\tilde{\mu}^{2}=\mu^{2} \frac{e^{\gamma_{E}}}{4 \pi}
$$

with $\gamma_{E} \simeq 0.577$ being Euler's constant. One-loop functions are summarized in section C.3.

\section{C.1 Scalar}

The Lagrangian density is given by ${ }^{14}$

$$
\mathcal{L}=-\frac{1}{4} F_{\mu \nu}^{2}-\frac{1}{2 \xi}\left(\partial_{\mu} A^{\mu}\right)^{2}+\left|D_{\mu} \phi\right|^{2}-m^{2}|\phi|^{2}-\frac{1}{4} \lambda|\phi|^{4},
$$

with $D_{\mu}=\partial_{\mu}-i q e A_{\mu}$ being the gauge covariant derivative for a charge $q$. We have integrated out the NL and (anti-)ghost fields. Parameters are a gauge coupling $e$, a scalar mass $m$, a quartic coupling $\lambda$, and a gauge fixing parameter $\xi$. Multiplicative renormalization is set for fields as $\phi=Z_{2}^{1 / 2} \bar{\phi}$ and $A_{\mu}=Z_{3}^{1 / 2} \bar{A}_{\mu}$ and for parameters as $Z_{2} Z_{3}^{1 / 2} e=Z_{1} \tilde{\mu}^{\epsilon / 2} \bar{e}$,

\footnotetext{
${ }^{14}$ The following procedure is simplified with the hep-th notation since $A_{\mu}$ has a mass dimension 1 and is not renormalized due to the Ward-Takahashi identity. In this case, one needs to multiply $e^{2}$ when translating $\mathcal{M}_{T A A}$ to $\mathcal{M}_{\text {dec }}$ since the gauge field is not canonically normalized.
} 


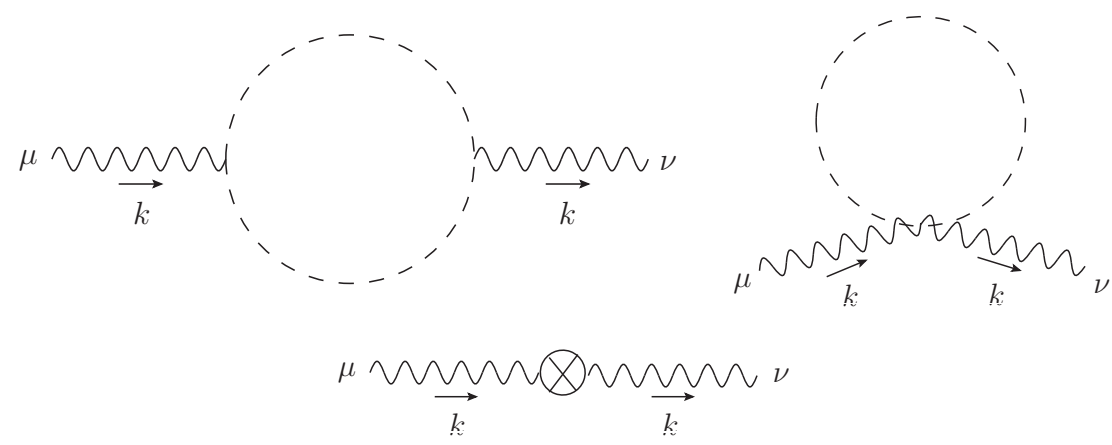

Figure 2. Leading contributions to the vacuum polarization $i \bar{\Pi}^{\mu \nu}$. [Top] scalar loop. [Bottom] counter term.

$Z_{2} Z_{3} e^{2}=Z_{4} \tilde{\mu}^{\epsilon} \bar{e}^{2}$ (i.e., $\left.Z_{2} Z_{4}=Z_{1}^{2}\right), Z_{2} m^{2}=Z_{m} \bar{m}^{2}, Z^{2} \lambda=Z_{\lambda} \tilde{\mu}^{\epsilon} \bar{\lambda}$, and $Z_{3} / \xi=Z_{5} / \bar{\xi}$. The Lagrangian density can be written in the form of renormalized perturbation theory as

$$
\begin{aligned}
\mathcal{L}= & -\frac{1}{4} \bar{F}_{\mu \nu}^{2}-\frac{1}{2 \bar{\xi}}\left(\partial_{\mu} \bar{A}^{\mu}\right)^{2}+\left|\partial_{\mu} \bar{\phi}\right|^{2}-\bar{m}^{2}|\bar{\phi}|^{2} \\
& -\frac{1}{4} Z_{\lambda} \tilde{\mu}^{\epsilon} \bar{\lambda}|\bar{\phi}|^{4}+i q Z_{1} \tilde{\mu}^{\epsilon / 2} \bar{e} \bar{A}^{\mu}\left(\bar{\phi}^{*} \partial_{\mu} \bar{\phi}-\partial_{\mu} \bar{\phi}^{*} \bar{\phi}\right)+q^{2} Z_{4} \tilde{\mu}^{\epsilon} \bar{e}^{2} \bar{A}_{\mu}^{2}|\bar{\phi}|^{2} \\
& -\frac{1}{4}\left(Z_{3}-1\right) \bar{F}_{\mu \nu}^{2}-\frac{1}{2 \bar{\xi}}\left(Z_{5}-1\right)\left(\partial_{\mu} \bar{A}^{\mu}\right)^{2}+\left(Z_{2}-1\right)\left|\partial_{\mu} \bar{\phi}\right|^{2}-\left(Z_{m}-1\right) \bar{m}^{2}|\bar{\phi}|^{2} .
\end{aligned}
$$

The Ward-Takahashi identity warrants that $Z_{1}=Z_{2}=Z_{4}, Z_{3}$ is independent of $\bar{\xi}$, and $Z_{5}=1$. It follows that

$$
\begin{aligned}
\beta_{e}^{\epsilon}= & -\frac{\bar{e}}{2} \epsilon\left(1-\frac{\bar{e}}{2} \frac{\partial \ln Z_{3}}{\partial \bar{e}}\right)^{-1}, \\
\beta_{\lambda}^{\epsilon}= & -\bar{\lambda} \epsilon\left(1-2 \bar{\lambda} \frac{\partial \ln Z_{2}}{\partial \bar{\lambda}}+\bar{\lambda} \frac{\partial \ln Z_{\lambda}}{\partial \bar{\lambda}}\right)^{-1}, \\
\beta_{m}= & \frac{\bar{m}}{2} \beta_{e}^{\epsilon}\left(\frac{\partial \ln Z_{2}}{\partial \bar{e}}-\frac{\partial \ln Z_{m}}{\partial \bar{e}}\right)+\frac{\bar{m}}{2} \beta_{\lambda}^{\epsilon}\left(\frac{\partial \ln Z_{2}}{\partial \bar{\lambda}}-\frac{\partial \ln Z_{m}}{\partial \bar{\lambda}}\right) \\
& +\frac{\bar{m}}{2} \beta_{\xi}\left(\frac{\partial \ln Z_{2}}{\partial \bar{\xi}}-\frac{\partial \ln Z_{m}}{\partial \bar{\xi}}\right), \\
\beta_{\xi}= & -\bar{\xi} \beta_{e}^{\epsilon} \frac{\partial \ln Z_{3}}{\partial \bar{e}}-\bar{\xi} \beta_{\lambda}^{\epsilon} \frac{\partial \ln Z_{2}}{\partial \bar{\lambda}} .
\end{aligned}
$$

$Z_{3}-1$ and $Z_{5}-1$ can be determined via loop corrections to the two point correlation function of the gauge boson:

$$
i \bar{\Pi}^{\mu \nu}=i \Pi^{\mu \nu}-i\left(Z_{3}-1\right)\left(k^{2} g^{\mu \nu}-k^{\mu} k^{\nu}\right)-i \frac{1}{\bar{\xi}}\left(Z_{5}-1\right) k^{\mu} k^{\nu},
$$

where $k$ denotes the gauge boson momentum. The one-loop vacuum polarization is given by (see the top diagrams of figure 2)

$$
\begin{aligned}
i \Pi^{\mu \nu} & =(i q \bar{e})^{2} i^{2} \tilde{\mu}^{\epsilon} \int \frac{d^{d} \ell}{(2 \pi)^{d}} \frac{(2 \ell+k)^{\mu}(2 \ell+k)^{\nu}}{\left[\ell^{2}-\bar{m}^{2}\right]\left[(\ell+k)^{2}-\bar{m}^{2}\right]}+\left(2 i q^{2} \bar{e}^{2} g^{\mu \nu}\right) i \tilde{\mu}^{\epsilon} \int \frac{d^{D} \ell}{(2 \pi)^{D}} \frac{1}{\left[\ell^{2}-\bar{m}^{2}\right]} \\
& =\frac{i q^{2} \bar{e}^{2}}{16 \pi^{2}}\left(\left[4 B_{22}-2 A\right] g^{\mu \nu}+\left[4 B_{21}+4 B_{1}+B_{0}\right] k^{\mu} k^{\nu}\right) .
\end{aligned}
$$


Noting that

$$
\begin{aligned}
4 B_{21}+4 B_{1}+B_{0} & =\frac{4}{3 k^{2}}\left[A-\bar{m}^{2} B_{0}+\frac{k^{2}}{4} B_{0}-\bar{m}^{2}+\frac{k^{2}}{6}\right] \\
& =-\frac{1}{k^{2}}\left[4 B_{22}-2 A\right],
\end{aligned}
$$

which ensures the Ward-Takahashi identity, one finds $i \Pi^{\mu \nu}=\left(k^{2} g^{\mu \nu}-k^{\mu} k^{\nu}\right) i \Pi$ and

$$
i \Pi=i \frac{4}{3 k^{2}} \frac{q^{2} \bar{e}^{2}}{16 \pi^{2}}\left[-A+\bar{m}^{2} B_{0}-\frac{k^{2}}{4} B_{0}+\bar{m}^{2}-\frac{k^{2}}{6}\right] .
$$

The pole of $\epsilon$ is canceled with (see the bottom diagram of figure 2)

$$
Z_{3}-1=-\frac{2}{3} \frac{q^{2} \bar{e}^{2}}{16 \pi^{2}} \frac{1}{\epsilon}
$$

and $Z_{5}-1=0$. Thus the four-dimension $\beta$ function is given by

$$
\beta_{e}=-\frac{\bar{e}^{2}}{4} \frac{\partial}{\partial \bar{e}}\left(\ln Z_{3}\right)_{\text {of } \epsilon=0}^{\text {residue }}=\frac{1}{3} \frac{q^{2} \bar{e}^{3}}{16 \pi^{2}} .
$$

The residue of the mass pole $\left(k^{2}=0\right)$ of the gauge field is given by

$$
Z_{3}^{\text {pole }}-1=\Pi-\left(Z_{3}-1\right)=\frac{1}{3} \frac{q^{2} \bar{e}^{2}}{16 \pi^{2}} \ln \left(\frac{\bar{m}^{2}}{\mu^{2}}\right) .
$$

The contribution from the one-loop diagram with the scalar mass term and $\eta$ term inserted is given by

$$
\begin{aligned}
\mathcal{M}_{|\phi|^{2}}= & 2\left(\bar{m}^{2}-\bar{\eta} k^{2}\right)\left((i q \bar{e})^{2} i^{3} \int \frac{d^{4} \ell}{(2 \pi)^{4}} \frac{\left(2 \ell+k_{1}\right) \cdot \epsilon_{1}^{*}\left(2 \ell+2 k_{1}+k_{2}\right) \cdot \epsilon_{2}^{*}}{\left[\ell^{2}\right]\left[\left(\ell+k_{1}\right)^{2}-\bar{m}^{2}\right]\left[\left(\ell+k_{1}+k_{2}\right)^{2}-\bar{m}^{2}\right]}\right. \\
& \left.+[1 \leftrightarrow 2]+2 i q^{2} \bar{e}^{2} \epsilon_{1}^{*} \cdot \epsilon_{2}^{*} i^{2} \int \frac{d^{4} \ell}{(2 \pi)^{4}} \frac{1}{\left[\ell^{2}-\bar{m}^{2}\right]\left[\left(\ell+k_{1}+k_{2}\right)^{2}-\bar{m}^{2}\right]}\right) \\
= & 2\left(\bar{m}^{2}-\bar{\eta} k^{2}\right) \frac{q^{2} \bar{e}^{2}}{16 \pi^{2}} \\
& \times\left(\left[-4 C_{24}+B_{0}\left(k^{2}\right)\right] \epsilon_{1}^{*} \cdot \epsilon_{2}^{*}+\left[-4 C_{23}-4 C_{12}\right] k_{2} \cdot \epsilon_{1}^{*} k_{1} \cdot \epsilon_{2}^{*}+[1 \leftrightarrow 2]\right) . \quad(\text { C. } 12)
\end{aligned}
$$

Noting that

$$
\begin{aligned}
-4 C_{23}-4 C_{12} & =\frac{1}{2 k^{2}}\left[2 m^{2} C_{0}+1\right] \\
& =-\frac{1}{2 k^{2}}\left[-4 C_{24}+B_{0}\left(k^{2}\right)\right],
\end{aligned}
$$

one finds

$$
\mathcal{M}_{|\phi|^{2}}=-4 \frac{\bar{m}^{2}-\bar{\eta} k^{2}}{k^{2}} \frac{q^{2} \bar{e}^{2}}{16 \pi^{2}}\left[2 \bar{m}^{2} C_{0}+1+[1 \leftrightarrow 2]\right]\left(k_{1} \cdot k_{2} \epsilon_{1}^{*} \cdot \epsilon_{2}^{*}-k_{2} \cdot \epsilon_{1}^{*} k_{1} \cdot \epsilon_{2}^{*}\right) .
$$

With

$$
2 \bar{m}^{2} C_{0}+1=-\frac{k^{2}}{12 \bar{m}^{2}} I_{s}\left(\frac{k^{2}}{\bar{m}^{2}}\right)
$$


the matrix element is

$$
\mathcal{M}_{|\phi|^{2}}=\frac{2}{3} \frac{q^{2} \bar{e}^{2}}{16 \pi^{2}} \frac{\bar{m}^{2}-\bar{\eta} k^{2}}{\bar{m}^{2}} I_{s}\left(\frac{k^{2}}{\bar{m}^{2}}\right)\left(k_{1} \cdot k_{2} \epsilon_{1}^{*} \cdot \epsilon_{2}^{*}-k_{2} \cdot \epsilon_{1}^{*} k_{1} \cdot \epsilon_{2}^{*}\right) .
$$

Let us see how we obtain the above result in Pauli-Villars regularization. Above the Pauli-Villars mass scale, $\beta_{e}=0$ and the trace of energy-momentum tensor is replaced by

$$
T_{\mu}^{\mu} \supset 2 \bar{m}^{2}|\bar{\phi}|^{2}+2 \bar{\eta} \partial^{2}|\bar{\phi}|^{2}+2 \bar{m}_{\mathrm{PV}}^{2}\left|\bar{\phi}_{\mathrm{PV}}\right|^{2}+2 \bar{\eta}_{\mathrm{PV}} \partial^{2}\left|\bar{\phi}_{\mathrm{PV}}\right|^{2},
$$

where $\bar{\phi}_{\mathrm{PV}}$ is a Pauli-Villars partner with a wrong statistics. As a result, the matrix element is replaced by

$$
\begin{aligned}
\mathcal{M}_{T A A}= & -\frac{2}{3} \frac{q^{2} \bar{e}^{2}}{16 \pi^{2}}\left(\frac{\bar{m}_{\mathrm{PV}}^{2}-\bar{\eta}_{\mathrm{PV}} k^{2}}{\bar{m}_{\mathrm{PV}}^{2}} I_{s}\left(\frac{k^{2}}{\bar{m}_{\mathrm{PV}}^{2}}\right)-\frac{\bar{m}^{2}-\bar{\eta} k^{2}}{\bar{m}^{2}} I_{s}\left(\frac{k^{2}}{\bar{m}^{2}}\right)\right) \\
& \times\left(k_{1} \cdot k_{2} \epsilon_{1}^{*} \cdot \epsilon_{2}^{*}-k_{1} \cdot \epsilon_{2}^{*} k_{1} \cdot \epsilon_{2}^{*}\right) .
\end{aligned}
$$

After integrating out the Pauli-Villars partner, i.e., $\bar{m}_{\mathrm{PV}}^{2} \rightarrow \infty$, one reproduces the above result.

\section{C.2 Fermion}

The Lagrangian density is given by

$$
\mathcal{L}=-\frac{1}{4} F_{\mu \nu}^{2}-\frac{1}{2 \xi}\left(\partial_{\mu} A^{\mu}\right)^{2}-\frac{1}{2} i D_{\mu} \bar{\psi} \gamma^{\mu} \psi+\frac{1}{2} \bar{\psi} \gamma^{\mu} i D_{\mu} \psi-m \bar{\psi} \psi
$$

with $D_{\mu}=\partial_{\mu}-i q e A_{\mu}$ being the gauge covariant derivative for a charge $q$. We have integrated out the Nakanishi-Lautrup and (anti-)ghost fields. Parameters are a gauge coupling $e$, a fermion mass $m$, and a gauge fixing parameter $\xi$.

Multiplicative renormalization is set for fields as $\psi=Z_{2}^{1 / 2} \bar{\psi},{ }^{15}$ and $A_{\mu}=Z_{3}^{1 / 2} \bar{A}_{\mu}$ and for parameters as $Z_{2} Z_{3}^{1 / 2} e=Z_{1} \tilde{\mu}^{\epsilon / 2} \bar{e}, Z_{2} m=Z_{m} \bar{m}$, and $Z_{3} / \xi=Z_{4} / \bar{\xi}$. The Lagrangian density can be written in the form of renormalized perturbation theory as

$$
\begin{aligned}
\mathcal{L}= & -\frac{1}{4} \bar{F}_{\mu \nu}^{2}-\frac{1}{2 \bar{\xi}}\left(\partial_{\mu} \bar{A}^{\mu}\right)^{2}-\frac{1}{2} i D_{\mu} \overline{\bar{\psi}} \gamma^{\mu} \bar{\psi}+\frac{1}{2} \bar{\psi} \gamma^{\mu} i D_{\mu} \bar{\psi}-\bar{m} \bar{\psi} \bar{\psi}+q Z_{1} \bar{e} \tilde{\mu}^{\epsilon / 2} \overline{A_{\mu}} \overline{\bar{\psi}} \gamma^{\mu} \bar{\psi} \\
& -\frac{1}{4}\left(Z_{3}-1\right) \bar{F}_{\mu \nu}^{2}-\frac{1}{2 \bar{\xi}}\left(Z_{4}-1\right)\left(\partial_{\mu} \bar{A}^{\mu}\right)^{2}-\left(Z_{2}-1\right) \frac{1}{2} i D_{\mu} \overline{\bar{\psi}} \gamma^{\mu} \bar{\psi} \\
& +\left(Z_{2}-1\right) \frac{1}{2} \bar{\psi} \gamma^{\mu} i D_{\mu} \bar{\psi}-\left(Z_{m}-1\right) \bar{m} \bar{\psi} \bar{\psi}
\end{aligned}
$$

The Ward-Takahashi identity warrants that $Z_{1}=Z_{2}, Z_{3}$ is independent of $\bar{\xi}$, and $Z_{4}=1$. It follows that

$$
\begin{aligned}
\beta_{e}^{\epsilon} & =-\frac{\bar{e}}{2} \epsilon\left(1-\frac{\bar{e}}{2} \frac{\partial \ln Z_{3}}{\partial \bar{e}}\right)^{-1}, \\
\beta_{m} & =\bar{m} \beta_{e}^{\epsilon}\left(\frac{\partial \ln Z_{2}}{\partial \bar{e}}-\frac{\partial \ln Z_{m}}{\partial \bar{e}}\right)+\bar{m} \beta_{\xi}\left(\frac{\partial \ln Z_{2}}{\partial \bar{\xi}}-\frac{\partial \ln Z_{m}}{\partial \bar{\xi}}\right) \\
\beta_{\xi} & =-\beta_{e}^{\epsilon} \frac{\partial \ln Z_{3}}{\partial \bar{e}} \bar{\xi}
\end{aligned}
$$




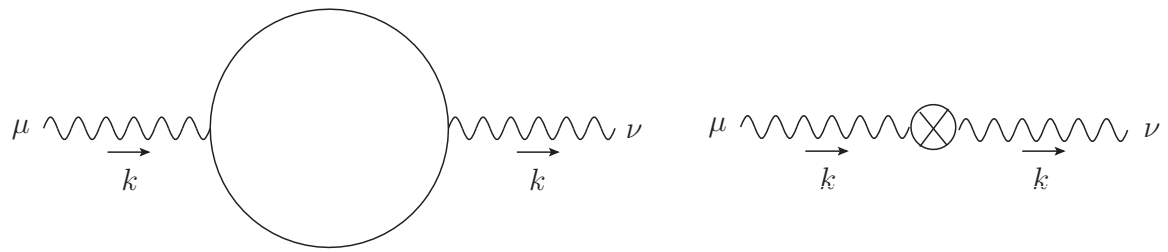

Figure 3. Leading contributions to the vacuum polarization $i \bar{\Pi}^{\mu \nu}$. [Left] fermion loop $i \Pi^{\mu \nu}$. [Right] counter term.

$Z_{3}-1$ and $Z_{4}-1$ can be determined via loop corrections to the two point correlation function of the gauge boson:

$$
i \bar{\Pi}^{\mu \nu}=i \Pi^{\mu \nu}-i\left(Z_{3}-1\right)\left(k^{2} g^{\mu \nu}-k^{\mu} k^{\nu}\right)-i \frac{1}{\bar{\xi}}\left(Z_{4}-1\right) k^{\mu} k^{\nu},
$$

where $k$ denotes the gauge boson momentum. The one-loop vacuum polarization is given by (see the left diagram of figure 3 )

$$
\begin{aligned}
i \Pi^{\mu \nu} & =(i q \bar{e})^{2}(-1) i^{2} \tilde{\mu}^{\epsilon} \int \frac{d^{d} \ell}{(2 \pi)^{d}} \frac{\operatorname{tr}\left[\gamma^{\mu}(\ell+\not k+\bar{m}) \gamma^{\nu}(\ell+\bar{m})\right]}{\left[\ell^{2}-\bar{m}^{2}\right]\left[(\ell+k)^{2}-\bar{m}^{2}\right]} \\
& =-\frac{i q^{2}}{16 \pi^{2}} 4\left(\left[(-2+\epsilon) B_{22}-k^{2}\left(B_{21}+B_{1}\right)+\bar{m}^{2} B_{0}\right] g^{\mu \nu}+\left[2 B_{21}+2 B_{1}\right] k^{\mu} k^{\nu}\right) .
\end{aligned}
$$

Noting that

$$
\begin{aligned}
2 B_{21}+2 B_{1} & =\frac{2}{3 k^{2}}\left[A-\bar{m}^{2} B_{0}-\frac{k^{2}}{2} B_{0}-\bar{m}^{2}+\frac{k^{2}}{6}\right] \\
& =-\frac{1}{k^{2}}\left[(-2+\epsilon) B_{22}-k^{2}\left(B_{21}+B_{1}\right)+\bar{m}^{2} B_{0}\right],
\end{aligned}
$$

which ensures the Ward-Takahashi identity, one finds $i \Pi^{\mu \nu}=\left(k^{2} g^{\mu \nu}-k^{\mu} k^{\nu}\right) i \Pi$ and

$$
i \Pi=i \frac{8}{3 k^{2}} \frac{q^{2} \bar{e}^{2}}{16 \pi^{2}}\left[A-\bar{m}^{2} B_{0}-\frac{k^{2}}{2} B_{0}-\bar{m}^{2}+\frac{k^{2}}{6}\right] .
$$

The pole at $\epsilon=0$ is canceled with (see the right diagram of figure 3)

$$
Z_{3}-1=-\frac{8}{3} \frac{q^{2} \bar{e}^{2}}{16 \pi^{2}} \frac{1}{\epsilon}
$$

and $Z_{4}-1=0$. Thus the four-dimension $\beta$ function is given by

$$
\beta_{e}=-\frac{\bar{e}^{2}}{4} \frac{\partial}{\partial \bar{e}}\left(\ln Z_{3}\right)_{\text {of } \epsilon=0}^{\text {residue }}=\frac{4}{3} \frac{q^{2} \bar{e}^{3}}{16 \pi^{2}} .
$$

The residue of the mass pole $\left(k^{2}=0\right)$ of the gauge field is given by

$$
Z_{3}^{\text {pole }}-1=\Pi-\left(Z_{3}-1\right)=\frac{4}{3} \frac{q^{2} \bar{e}^{2}}{16 \pi^{2}} \ln \left(\frac{\bar{m}^{2}}{\mu^{2}}\right) .
$$

\footnotetext{
${ }^{15}$ Note that a bar for a renormalized quantity is different from an overline for a Dirac bar.
} 
$d$-dimension flat-spacetime energy-momentum tensor is given by ${ }^{16}$

$$
\begin{aligned}
T_{\mu \nu}= & -g^{\lambda \kappa} F_{\mu \lambda} F_{\nu \kappa}-\frac{1}{4}\left(i D_{\mu} \bar{\psi} \gamma_{\nu}+i D_{\nu} \bar{\psi} \gamma_{\mu}\right) \psi+\frac{1}{4} \bar{\psi}\left(i D_{\mu} \gamma_{\nu}+i D_{\nu} \gamma_{\mu}\right) \psi \\
& -g_{\mu \nu}\left(-\frac{1}{4} F_{\mu \nu}^{2}-\frac{1}{2} i D_{\mu} \bar{\psi} \gamma^{\mu} \psi+\frac{1}{2} \bar{\psi} \gamma^{\mu} i D_{\mu} \psi-m \bar{\psi} \psi\right) .
\end{aligned}
$$

Taking a classical trace, one finds

$$
\left(T_{\mu}^{\mu}\right)_{\text {class }}=-\frac{1}{4} \epsilon F_{\mu \nu}^{2}+m \bar{\psi} \psi+\left(\frac{3}{2}-\frac{\epsilon}{2}\right)(\text { e.o.m })
$$

where

$$
\text { (e.o.m.) }=-(-i \not \supset \bar{\psi}-m \bar{\psi}) \psi-\bar{\psi}(i \not D-m) \psi .
$$

The first term of $\left(T_{\mu}^{\mu}\right)_{\text {class }}$ vanishes at the classical level as $\epsilon \rightarrow 0$, but not at the quantum level. This contribution provides $A_{\text {anom }}$. The leading contribution to $\mathcal{M}_{T A A}$ arises from the following trace of energy-momentum tensor:

$$
T_{\mu}^{\mu} \supset \frac{2}{3} \frac{q^{2} \bar{e}^{2}}{16 \pi^{2}} \bar{F}_{\mu \nu}^{2}+\bar{m} \overline{\bar{\psi}} \bar{\psi}
$$

The first term arises from the gauge kinetic term proportional to $\epsilon$ in eq. (C.30). Its coefficient is obtained from the leading contribution to the wave function renormalization $Z_{3}$ of the gauge field [see eq. (C.26)]. Note that the leading contribution to $Z_{3}$ also determines the leading contribution to the $\beta$ function $\beta_{e}$ [see eq. (C.27)] The all-order form that is often quoted,

$$
T_{\mu}^{\mu}=\frac{\beta_{e}}{2 \bar{e}}\left[\bar{F}_{\mu \nu}^{2}\right]+\left(\bar{m}-\beta_{m}\right)[\overline{\bar{\psi}} \bar{\psi}]
$$

is obtained after renormalization of composite operators [65].

The matrix element is

$$
\mathcal{M}_{T A A}=\mathcal{M}_{F^{2}}+\mathcal{M}_{\bar{\psi} \psi} .
$$

The first term arises from the tree-level diagram with the gauge kinetic term inserted (see the left diagram of figure 4):

$$
\mathcal{M}_{F^{2}}=-\frac{8}{3} \frac{q^{2} \bar{e}^{2}}{16 \pi^{2}}\left(k_{1} \cdot k_{2} \epsilon_{1}^{*} \cdot \epsilon_{2}^{*}-k_{2} \cdot \epsilon_{1}^{*} k_{1} \cdot \epsilon_{2}^{*}\right) .
$$

The second term is a one-loop contribution from the fermion mass term inserted (see the right diagram of figure 4$)$ :

$$
\begin{aligned}
\mathcal{M}_{\bar{\psi} \psi}= & \bar{m}\left((i q \bar{e})^{2}(-1) i^{3} \int \frac{d^{4} \ell}{(2 \pi)^{4}} \frac{\operatorname{tr}\left[\left(\ell+\not k_{1}+\not k_{2}+\bar{m}\right) \notin_{2}^{*}\left(\ell+\not k_{1}+\bar{m}\right) \notin_{1}^{*}(\ell+\bar{m})\right]}{\left[\ell^{2}-\bar{m}^{2}\right]\left[\left(\ell+k_{1}\right)^{2}-\bar{m}^{2}\right]\left[\left(\ell+k_{1}+k_{2}\right)^{2}-\bar{m}^{2}\right]}+[1 \leftrightarrow 2]\right) \\
= & 4 \bar{m}^{2} \frac{q^{2} \bar{e}^{2}}{16 \pi^{2} \tilde{\mu}^{\epsilon}}\left(\left[\epsilon C_{24}-k^{2} C_{23}-k^{2} C_{12}-\frac{k^{2}}{2} C_{0}+\bar{m}^{2} C_{0}\right] \epsilon_{1}^{*} \cdot \epsilon_{2}^{*}\right. \\
& \left.+\left[4 C_{23}+4 C_{12}+C_{0}\right] k_{2} \cdot \epsilon_{1}^{*} k_{1} \cdot \epsilon_{2}^{*}+[1 \leftrightarrow 2]\right) .
\end{aligned}
$$

\footnotetext{
${ }^{16}$ Curved-spacetime energy-momentum tensor takes the same form with $D_{\mu}$ being the gauge, Local Lorentz, and diffeomorphism covariant derivative.
} 

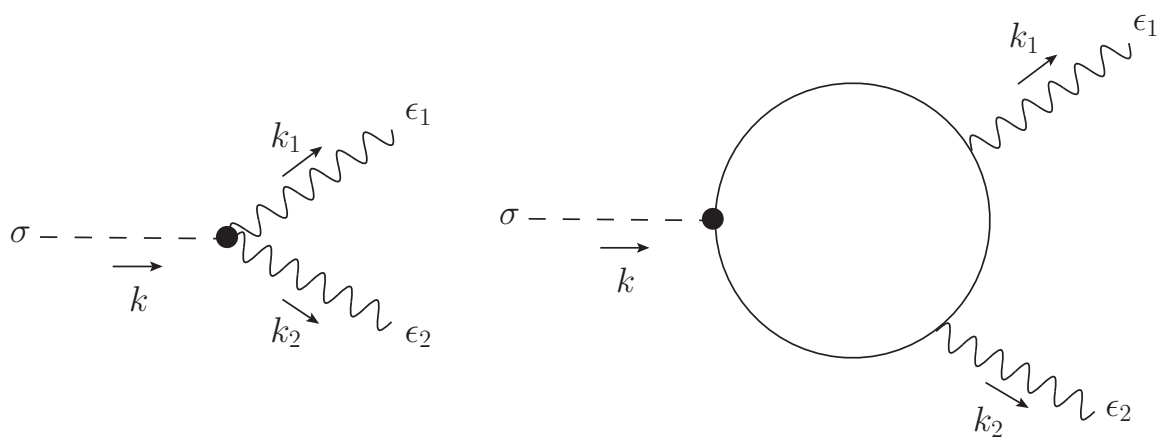

Figure 4. Leading contributions to $\mathcal{M}_{T A A}$, i.e., $T_{\mu}^{\mu}-\bar{A}_{\lambda}-\bar{A}_{\kappa}$ correlation function ( $\sigma$ decay into two gauge bosons). [Left] $\mathcal{M}_{F^{2}}$ : the gauge kinetic term in eq. (C.33) is inserted. [Right] $\mathcal{M}_{\bar{\psi} \psi}$ : the fermion mass term in eq. (C.33) is inserted. There is the other contribution with the external gauge bosons exchanged.

Noting that

$$
\begin{aligned}
4 C_{23}+4 C_{12}+C_{0} & =-\frac{1}{2 k^{2}}\left[2 \bar{m}^{2} C_{0}+1-\frac{k^{2}}{2} C_{0}\right] \\
& =-\frac{1}{2 k^{2}}\left[\epsilon C_{24}-k^{2} C_{23}-k^{2} C_{12}-\frac{k^{2}}{2} C_{0}+\bar{m}^{2} C_{0}\right],
\end{aligned}
$$

one finds

$$
\mathcal{M}_{\bar{\psi} \psi}=8 \frac{\bar{m}^{2}}{k^{2}} \frac{q^{2} \bar{e}^{2}}{16 \pi^{2}}\left[2 \bar{m}^{2} C_{0}+1-\frac{k^{2}}{2} C_{0}+[1 \leftrightarrow 2]\right]\left(k_{1} \cdot k_{2} \epsilon_{1}^{*} \cdot \epsilon_{2}^{*}-k_{2} \cdot \epsilon_{1}^{*} k_{1} \cdot \epsilon_{2}^{*}\right)
$$

With

$$
2 \bar{m}^{2} C_{0}+1-\frac{k^{2}}{2} C_{0}=\frac{k^{2}}{6 \bar{m}^{2}} I_{f}\left(\frac{k^{2}}{m^{2}}\right)
$$

the matrix element is

$$
\mathcal{M}_{\bar{\psi} \psi}=\frac{8}{3} \frac{q^{2} \bar{e}^{2}}{16 \pi^{2}} I_{f}\left(\frac{k^{2}}{\bar{m}^{2}}\right)\left(k_{1} \cdot k_{2} \epsilon_{1}^{*} \cdot \epsilon_{2}^{*}-k_{2} \cdot \epsilon_{1}^{*} k_{1} \cdot \epsilon_{2}^{*}\right) .
$$

Collecting the two contributions, one obtains

$$
\mathcal{M}_{T A A}=\frac{8}{3} \frac{q^{2} \bar{e}^{2}}{16 \pi^{2}}\left(1-I_{f}\left(\frac{k^{2}}{\bar{m}^{2}}\right)\right)\left(k_{1} \cdot k_{2} \epsilon_{1}^{*} \cdot \epsilon_{2}^{*}-k_{2} \cdot \epsilon_{1}^{*} k_{1} \cdot \epsilon_{2}^{*}\right)
$$

We remark that $I_{f}(0)=1$ and thus a heavy $\left(\bar{m}^{2} \gg k^{2}\right)$ fermion does not contribute to $\mathcal{M}_{T A A}$. Meanwhile, $I_{f}(\infty)=0$ and thus a light $\left(\bar{m}^{2} \ll k^{2}\right)$ fermion indeed contributes to $\mathcal{M}_{T A A}$.

Let us see how we obtain the above result in Pauli-Villars regularization. Above the Pauli-Villars mass scale, $\beta_{e}=0$ and the trace of energy-momentum tensor is replaced by

$$
T_{\mu}^{\mu} \supset \bar{m} \bar{\psi} \bar{\psi}+\bar{m}_{\mathrm{PV}} \overline{\bar{\psi}}_{\mathrm{PV}} \bar{\psi}_{\mathrm{PV}}
$$


where $\bar{\psi}_{\mathrm{PV}}$ is a Pauli-Villars partner with a wrong statistics. As a result, the matrix element is replaced by

$$
\mathcal{M}_{T A A}=\frac{8}{3} \frac{q^{2} \bar{e}^{2}}{16 \pi^{2}}\left(I_{f}\left(\frac{k^{2}}{\bar{m}_{\mathrm{PV}}^{2}}\right)-I_{f}\left(\frac{k^{2}}{\bar{m}^{2}}\right)\right)\left(k_{1} \cdot k_{2} \epsilon_{1}^{*} \cdot \epsilon_{2}^{*}-k_{2} \cdot \epsilon_{1}^{*} k_{1} \cdot \epsilon_{2}^{*}\right) .
$$

After integrating out the Pauli-Villars partner, i.e., $\bar{m}_{\mathrm{PV}}^{2} \rightarrow \infty$, one reproduces the above result.

\section{C.3 Summary of one-loop functions}

One-loop functions are based on refs. [117, 118] (see also appendix F of ref. [119]). One point integral is defined as

$$
\tilde{\mu}^{\epsilon} \int \frac{d^{d} \ell}{(2 \pi)^{d}} \frac{1}{\ell^{2}-m^{2}}=\frac{i}{16 \pi^{2}} A\left(m^{2}\right) .
$$

The explicit form is

$$
A\left(m^{2}\right)=m^{2}\left(\frac{2}{\epsilon}-\ln \left(\frac{m^{2}}{\mu^{2}}\right)+1\right) .
$$

Two point integrals are defined as

$$
\tilde{\mu}^{\epsilon} \int \frac{d^{d} \ell}{(2 \pi)^{d}} \frac{1 ; \ell_{\mu} ; \ell_{\mu} \ell_{\nu}}{\left[\ell^{2}-m_{1}^{2}\right]\left[(\ell+k)^{2}-m_{2}^{2}\right]}=\frac{i}{16 \pi^{2}} B_{0 ; \mu ; \mu \nu}\left(k^{2} ; m_{1}^{2}, m_{2}^{2}\right),
$$

where

$$
\begin{aligned}
& B_{\mu}=k_{\mu} B_{1}, \\
& B_{\mu \nu}=g_{\mu \nu} B_{22}+k_{\mu} k_{\nu} B_{21} .
\end{aligned}
$$

For our purpose, we can take $m_{1}=m_{2}=m$ :

$$
\begin{aligned}
B_{1} & =-\frac{1}{2} B_{0}, \\
B_{22} & =\frac{1}{6}\left[A+2 \bar{m}^{2} B_{0}-\frac{k^{2}}{2} B_{0}+2 \bar{m}^{2}-\frac{k^{2}}{3}\right], \\
B_{21} & =\frac{1}{3 k^{2}}\left[A-\bar{m}^{2} B_{0}+k^{2} B_{0}-\bar{m}^{2}+\frac{k^{2}}{6}\right] .
\end{aligned}
$$

The explicit form with a Feynman parameter integral is

$$
B_{0}=\frac{2}{\epsilon}-\int_{0}^{1} d x \ln \left(\frac{m^{2}-x(1-x) k^{2}-i \epsilon_{\mathrm{ad}}}{\mu^{2}}\right) .
$$

Three point integrals are defined as

$$
\begin{aligned}
\tilde{\mu}^{\epsilon} \int \frac{d^{d} \ell}{(2 \pi)^{d}} \frac{1 ; \ell_{\mu} ; \ell_{\mu} \ell_{\nu}}{\left[\ell^{2}-m_{1}^{2}\right]\left[\left(\ell+k_{1}\right)^{2}-m_{2}^{2}\right]\left[\left(\ell+k_{1}+k_{2}\right)^{2}-m_{3}^{2}\right]} \\
=\frac{i}{16 \pi^{2}} C_{0 ; \mu ; \mu \nu}\left(k_{1}^{2}, k_{2}^{2}, k^{2} ; m_{1}^{2}, m_{2}^{2}, m_{3}^{2}\right),
\end{aligned}
$$


where $k+k_{1}+k_{2}=0$ and

$$
\begin{aligned}
C_{\mu} & =k_{1 \mu} C_{11}+k_{2 \mu} C_{12}, \\
C_{\mu \nu} & =g_{\mu \nu} C_{24}+k_{1 \mu} k_{1 \nu} C_{21}+k_{2 \mu} k_{2 \nu} C_{22}+\left(k_{1 \mu} k_{2 \nu}+k_{2 \mu} k_{1 \nu}\right) C_{23} .
\end{aligned}
$$

For our purpose, again we can take $m_{1}=m_{2}=m_{3}=m$ :

$$
\begin{aligned}
& C_{11}=\frac{1}{k^{2}}\left[B_{0}\left(k_{1}^{2}\right)-B_{0}\left(k^{2}\right)-k^{2} C_{0}\right], \\
& C_{12}=\frac{1}{k^{2}}\left[B_{0}\left(k^{2}\right)-B_{0}\left(k_{2}^{2}\right)\right], \\
& C_{24}=\frac{1}{4}\left[B_{0}\left(k^{2}\right)+2 \bar{m}^{2} C_{0}+1\right], \\
& C_{21}=-\frac{1}{2 k^{2}}\left[3 B_{0}\left(k^{2}\right)-3 B_{0}\left(k^{2}\right)-2 k^{2} C_{0}\right], \\
& C_{23}=-\frac{1}{2 k^{2}}\left[2 B_{0}\left(k^{2}\right)-2 B_{0}\left(k_{2}^{2}\right)+2 \bar{m}^{2} C_{0}+1\right], \\
& C_{22}=-\frac{1}{2 k^{2}}\left[B_{0}\left(k^{2}\right)-B_{0}\left(k_{2}^{2}\right)\right] .
\end{aligned}
$$

The explicit form with Feynman parameter integrals is

$$
C_{0}=-\int_{0}^{1} d x \int_{0}^{1-x} d y \frac{1}{-k^{2} x y+m^{2}-i \epsilon_{\mathrm{ad}}} .
$$

Open Access. This article is distributed under the terms of the Creative Commons Attribution License (CC-BY 4.0), which permits any use, distribution and reproduction in any medium, provided the original author(s) and source are credited.

\section{References}

[1] R. Brout, F. Englert and E. Gunzig, The creation of the Universe as a quantum phenomenon, Annals Phys. 115 (1978) 78 [INSPIRE].

[2] A.A. Starobinsky, A new type of isotropic cosmological models without singularity, Phys. Lett. B 91 (1980) 99 [INSPIRE].

[3] D. Kazanas, Dynamics of the Universe and spontaneous symmetry breaking, Astrophys. J. 241 (1980) L59 [INSPIRE].

[4] A.H. Guth, The inflationary Universe: a possible solution to the horizon and flatness problems, Phys. Rev. D 23 (1981) 347 [INSPIRE].

[5] K. Sato, First order phase transition of a vacuum and expansion of the universe, Mon. Not. Roy. Astron. Soc. 195 (1981) 467 [inSPIRE].

[6] A.D. Linde, A new inflationary universe scenario: a possible solution of the horizon, flatness, homogeneity, isotropy and primordial monopole problems, Phys. Lett. B 108 (1982) 389 [INSPIRE].

[7] A. Albrecht and P.J. Steinhardt, Cosmology for grand unified theories with radiatively induced symmetry breaking, Phys. Rev. Lett. 48 (1982) 1220 [INSPIRE].

[8] A.D. Linde, Chaotic inflation, Phys. Lett. B 129 (1983) 177 [InSPIRE]. 
[9] V.F. Mukhanov and G.V. Chibisov, Quantum fluctuations and a nonsingular universe, JETP Lett. 33 (1981) 532 [INSPIRE].

[10] V.F. Mukhanov and G.V. Chibisov, The vacuum energy and large scale structure of the universe, Sov. Phys. JETP 56 (1982) 258 [INSPIRE].

[11] S.W. Hawking, The development of irregularities in a single bubble inflationary universe, Phys. Lett. B 115 (1982) 295 [INSPIRE].

[12] A.H. Guth and S.Y. Pi, Fluctuations in the new inflationary universe, Phys. Rev. Lett. 49 (1982) 1110 [INSPIRE].

[13] A.A. Starobinsky, Dynamics of phase transition in the new inflationary universe scenario and generation of perturbations, Phys. Lett. B 117 (1982) 175 [INSPIRE].

[14] J.M. Bardeen, P.J. Steinhardt and M.S. Turner, Spontaneous creation of almost scale-free density perturbations in an inflationary universe, Phys. Rev. D 28 (1983) 679 [INSPIRE].

[15] V.F. Mukhanov, Gravitational instability of the universe filled with a scalar field, JETP Lett. 41 (1985) 493 [INSPIRE].

[16] PLanck collaboration, Planck 2018 results. X. Constraints on inflation, arXiv: 1807.06211 [INSPIRE].

[17] J. Martin, C. Ringeval and V. Vennin, Encyclopæedia inflationaris, Phys. Dark Univ. 5-6 (2014) 75 [arXiv:1303.3787] [INSPIRE].

[18] J.D. Barrow and A.C. Ottewill, The stability of general relativistic cosmological theory, J. Phys. A 16 (1983) 2757 [inSPIRE].

[19] B. Whitt, Fourth order gravity as general relativity plus matter, Phys. Lett. B 145 (1984) 176 [INSPIRE].

[20] A. Vilenkin, Classical and quantum cosmology of the Starobinsky inflationary model, Phys. Rev. D 32 (1985) 2511 [INSPIRE].

[21] M.B. Mijić, M.S. Morris and W.-M. Suen, The $R^{2}$ cosmology: inflation without a phase transition, Phys. Rev. D 34 (1986) 2934 [inSPIRE].

[22] J.D. Barrow and S. Cotsakis, Inflation and the conformal structure of higher order gravity theories, Phys. Lett. B 214 (1988) 515 [INSPIRE].

[23] T. Matsumura et al., LiteBIRD: mission overview and focal plane layout, J. Low. Temp. Phys. 184 (2016) 824 [INSPIRE].

[24] CMB-S4 collaboration, CMB-S4 science book, first edition, arXiv:1610.02743 [INSPIRE].

[25] CORE collaboration, Exploring cosmic origins with CORE: Survey requirements and mission design, JCAP 04 (2018) 014 [arXiv:1706.04516] [INSPIRE].

[26] F.L. Bezrukov and D.S. Gorbunov, Distinguishing between $R^{2}$-inflation and Higgs-inflation, Phys. Lett. B 713 (2012) 365 [arXiv:1111.4397] [INSPIRE].

[27] D.S. Gorbunov and A.G. Panin, Scalaron the mighty: producing dark matter and baryon asymmetry at reheating, Phys. Lett. B 700 (2011) 157 [arXiv: 1009.2448] [INSPIRE].

[28] D.S. Gorbunov and A.G. Panin, Free scalar dark matter candidates in $R^{2}$-inflation: the light, the heavy and the superheavy, Phys. Lett. B 718 (2012) 15 [arXiv:1201.3539] [INSPIRE]. 
[29] W. Buchmüller, P. Di Bari and M. Plümacher, Cosmic microwave background, matter-antimatter asymmetry and neutrino masses, Nucl. Phys. B 643 (2002) 367 [Erratum ibid. B 793 (2008) 362] [hep-ph/0205349] [INSPIRE].

[30] G.F. Giudicee $\mathrm{t}$ al., Towards a complete theory of thermal leptogenesis in the SM and MSSM, Nucl. Phys. B 685 (2004) 89 [hep-ph/0310123] [InSPIRE].

[31] W. Buchmüller, R.D. Peccei and T. Yanagida, Leptogenesis as the origin of matter, Ann. Rev. Nucl. Part. Sci. 55 (2005) 311 [hep-ph/0502169] [InSPIRE].

[32] S. Davidson, E. Nardi and Y. Nir, Leptogenesis, Phys. Rept. 466 (2008) 105 [arXiv:0802.2962] [INSPIRE].

[33] M. Fukugita and T. Yanagida, Baryogenesis without grand unification, Phys. Lett. B 174 (1986) 45 [INSPIRE].

[34] D. Gorbunov and A. Tokareva, $R^{2}$-inflation with conformal SM Higgs field, JCAP 12 (2013) 021 [arXiv: 1212.4466] [INSPIRE].

[35] S. Kawamura et al., The Japanese space gravitational wave antenna DECIGO, Class. Quant. Grav. 23 (2006) S125 [INSPIRE].

[36] A. Jakubiec and J. Kijowski, On theories of gravitation with nonlinear lagrangians, Phys. Rev. D 37 (1988) 1406 [INSPIRE].

[37] T. Faulkner, M. Tegmark, E.F. Bunn and Y. Mao, Constraining $f(R)$ gravity as a scalar tensor theory, Phys. Rev. D 76 (2007) 063505 [astro-ph/0612569] [INSPIRE].

[38] F.S. Accetta, D.J. Zoller and M.S. Turner, Induced gravity inflation, Phys. Rev. D 31 (1985) 3046 [INSPIRE].

[39] D. La and P.J. Steinhardt, Extended inflationary cosmology, Phys. Rev. Lett. 62 (1989) 376 [Erratum ibid. 62 (1989) 1066] [INSPIRE].

[40] T. Futamase and K.-i. Maeda, Chaotic inflationary scenario of the Universe with a nonminimally coupled "inflaton" field, Phys. Rev. D 39 (1989) 399 [INSPIRE].

[41] D.S. Salopek, J.R. Bond and J.M. Bardeen, Designing density fluctuation spectra in inflation, Phys. Rev. D 40 (1989) 1753 [inSPIRE].

[42] R. Fakir and W.G. Unruh, Improvement on cosmological chaotic inflation through nonminimal coupling, Phys. Rev. D 41 (1990) 1783 [INSPIRE].

[43] K. Kannike et al., Dynamically induced Planck scale and inflation, JHEP 05 (2015) 065 [arXiv: 1502.01334] [INSPIRE].

[44] S.-M. Choi, Y.-J. Kang, H.M. Lee and K. Yamashita, Unitary inflaton as decaying dark matter, JHEP 05 (2019) 060 [arXiv: 1902.03781] [INSPIRE].

[45] J.R. Ellis, M.K. Gaillard and D.V. Nanopoulos, A phenomenological profile of the Higgs boson, Nucl. Phys. B 106 (1976) 292 [InSPIRE].

[46] M.A. Shifman, A.I. Vainshtein, M.B. Voloshin and V.I. Zakharov, Low-energy theorems for Higgs boson couplings to photons, Sov. J. Nucl. Phys. 30 (1979) 711 [INSPIRE].

[47] R.D. Peccei and H.R. Quinn, CP conservation in the presence of pseudoparticles, Phys. Rev. Lett. 38 (1977) 1440 [INSPIRE].

[48] R.D. Peccei and H.R. Quinn, Constraints imposed by CP conservation in the presence of pseudoparticles, Phys. Rev. D 16 (1977) 1791 [InSPIRE]. 
[49] S. Weinberg, A new light boson?, Phys. Rev. Lett. 40 (1978) 223 [InSPIRE].

[50] F. Wilczek, Problem of strong $P$ and $T$ invariance in the presence of instantons, Phys. Rev. Lett. 40 (1978) 279 [INSPIRE].

[51] J.E. Kim, Weak interaction singlet and strong CP invariance, Phys. Rev. Lett. 43 (1979) 103 [INSPIRE].

[52] M.A. Shifman, A.I. Vainshtein and V.I. Zakharov, Can confinement ensure natural CP invariance of strong interactions?, Nucl. Phys. B 166 (1980) 493 [InSPIRE].

[53] A.R. Zhitnitsky, On possible suppression of the axion hadron interactions (in Russian), Sov. J. Nucl. Phys. 31 (1980) 260 [inSPIRE].

[54] M. Dine, W. Fischler and M. Srednicki, A simple solution to the strong CP problem with a harmless axion, Phys. Lett. 104B (1981) 199 [INSPIRE].

[55] N. Takeda and Y. Watanabe, No quasistable scalaron lump forms after $R^{2}$ inflation, Phys. Rev. D 90 (2014) 023519 [arXiv: 1405.3830] [InSPIRE].

[56] Y. Watanabe, Rate of gravitational inflaton decay via gauge trace anomaly, Phys. Rev. D 83 (2011) 043511 [arXiv: 1011.3348] [INSPIRE].

[57] T. Katsuragawa and S. Matsuzaki, Dark matter in modified gravity?, Phys. Rev. D 95 (2017) 044040 [arXiv: 1610.01016] [inSPIRE].

[58] K. Falls and M. Herrero-Valea, Frame (in)equivalence in quantum field theory and cosmology, Eur. Phys. J. C 79 (2019) 595 [arXiv:1812.08187] [INSPIRE].

[59] C.G. Callan Jr., S.R. Coleman and R. Jackiw, A new improved energy-momentum tensor, Annals Phys. 59 (1970) 42 [InSPIRE].

[60] S.R. Coleman and R. Jackiw, Why dilatation generators do not generate dilatations?, Annals Phys. 67 (1971) 552 [INSPIRE].

[61] D.Z. Freedman, I.J. Muzinich and E.J. Weinberg, On the energy-momentum tensor in gauge field theories, Annals Phys. 87 (1974) 95 [INSPIRE].

[62] D.Z. Freedman and E.J. Weinberg, The energy-momentum tensor in scalar and gauge field theories, Annals Phys. 87 (1974) 354 [InSPIRE].

[63] J.C. Collins, Renormalization of the energy-momentum tensor in $\phi^{4}$ theory, Phys. Rev. D 14 (1976) 1965 [INSPIRE].

[64] N.K. Nielsen, The energy momentum tensor in a nonabelian quark gluon theory, Nucl. Phys. B 120 (1977) 212 [InSPIRE].

[65] S.L. Adler, J.C. Collins and A. Duncan, Energy-momentum-tensor trace anomaly in spin 1/2 quantum electrodynamics, Phys. Rev. D 15 (1977) 1712 [INSPIRE].

[66] J.C. Collins, A. Duncan and S.D. Joglekar, Trace and dilatation anomalies in gauge theories, Phys. Rev. D 16 (1977) 438 [InSPIRE].

[67] L.S. Brown, Dimensional regularization of composite operators in scalar field theory, Annals Phys. 126 (1980) 135 [INSPIRE].

[68] L.S. Brown and J.C. Collins, Dimensional renormalization of scalar field theory in curved space-time, Annals Phys. 130 (1980) 215 [INSPIRE].

[69] S.J. Hathrell, Trace anomalies and $\lambda \phi^{4}$ theory in curved space, Annals Phys. 139 (1982) 136 [INSPIRE]. 
[70] S.J. Hathrell, Trace anomalies and QED in curved space, Annals Phys. 142 (1982) 34 [INSPIRE].

[71] D.M. Capper, M.J. Duff and L. Halpern, Photon corrections to the graviton propagator, Phys. Rev. D 10 (1974) 461 [inSPIRE].

[72] S. Deser and P. van Nieuwenhuizen, One loop divergences of quantized Einstein-Maxwell fields, Phys. Rev. D 10 (1974) 401 [inSPIRE].

[73] D.M. Capper and M.J. Duff, Trace anomalies in dimensional regularization, Nuovo Cim. A 23 (1974) 173 [INSPIRE].

[74] D.M. Capper and M.J. Duff, The one loop neutrino contribution to the graviton propagator, Nucl. Phys. B 82 (1974) 147 [inSPIRE].

[75] M.J. Duff, Observations on conformal anomalies, Nucl. Phys. B 125 (1977) 334 [InSPIRE].

[76] J.S. Dowker and R. Critchley, Stress-tensor conformal anomaly for scalar, spinor, and vector fields, Phys. Rev. D 16 (1977) 3390 [inSPIRE].

[77] L.S. Brown, Stress tensor trace anomaly in a gravitational metric: scalar fields, Phys. Rev. D 15 (1977) 1469 [INSPIRE].

[78] S.M. Christensen and S.A. Fulling, Trace anomalies and the Hawking effect, Phys. Rev. D 15 (1977) 2088 [INSPIRE].

[79] L.S. Brown and J.P. Cassidy, Stress tensor trace anomaly in a gravitational metric: general theory, Maxwell field, Phys. Rev. D 15 (1977) 2810 [InSPIRE].

[80] T.S. Bunch and P.C.W. Davies, Quantum field theory in de Sitter space: renormalization by point splitting, Proc. Roy. Soc. Lond. A 360 (1978) 117 [INSPIRE].

[81] M.J. Duff, Twenty years of the Weyl anomaly, Class. Quant. Grav. 11 (1994) 1387 [hep-th/9308075] [INSPIRE].

[82] L. Randall and R. Sundrum, Out of this world supersymmetry breaking, Nucl. Phys. B 557 (1999) 79 [hep-th/9810155] [INSPIRE].

[83] G.F. Giudice, R. Rattazzi, M.A. Luty and H. Murayama, Gaugino mass without singlets, JHEP 12 (1998) 027 [hep-ph/9810442] [INSPIRE].

[84] M. Endo, F. Takahashi and T.T. Yanagida, Anomaly-induced inflaton decay and gravitino-overproduction problem, Phys. Lett. B 658 (2008) 236 [hep-ph/0701042] [INSPIRE].

[85] M. Endo, F. Takahashi and T.T. Yanagida, Inflaton decay in supergravity, Phys. Rev. D 76 (2007) 083509 [arXiv:0706.0986] [INSPIRE].

[86] T. Terada, Y. Watanabe, Y. Yamada and J. Yokoyama, Reheating processes after Starobinsky inflation in old-minimal supergravity, JHEP 02 (2015) 105 [arXiv:1411.6746] [INSPIRE].

[87] K. Fujikawa, Comment on chiral and conformal anomalies, Phys. Rev. Lett. 44 (1980) 1733 [INSPIRE].

[88] K. Fujikawa, Energy-momentum tensor in quantum field theory, Phys. Rev. D 23 (1981) 2262 [INSPIRE].

[89] K. Fujikawa, A nondiagramatic calculation of one loop $\beta$-function in $Q C D$, Phys. Rev. D 48 (1993) 3922 [INSPIRE]. 
[90] K. Fujikawa and H. Suzuki, Path integrals and quantum anomalies, Oxford University Press, Oxford U.K. (2004) [InSPIRE].

[91] J.F. Ashmore, A method of gauge invariant regularization, Lett. Nuovo Cim. 4 (1972) 289 [INSPIRE].

[92] C.G. Bollini and J.J. Giambiagi, Dimensional renormalization: the number of dimensions as a regularizing parameter, Nuovo Cim. B 12 (1972) 20 [INSPIRE].

[93] G. 't Hooft and M.J.G. Veltman, Regularization and renormalization of gauge fields, Nucl. Phys. B 44 (1972) 189 [INSPIRE].

[94] E.W. Kolb and M.S. Turner, The early universe, Front. Phys. 69 (1990) 1 [InSPIRE].

[95] K. Fujikawa, Path integral for gauge theories with fermions, Phys. Rev. D 21 (1980) 2848 [Erratum ibid. D 22 (1980) 1499] [INSPIRE].

[96] S.L. Adler and W.A. Bardeen, Absence of higher order corrections in the anomalous axial vector divergence equation, Phys. Rev. 182 (1969) 1517 [INSPIRE].

[97] W.A. Bardeen, Anomalous Ward identities in spinor field theories, Phys. Rev. 184 (1969) 1848 [INSPIRE].

[98] K. Yonekura, Notes on operator equations of supercurrent multiplets and anomaly puzzle in supersymmetric field theories, JHEP 09 (2010) 049 [arXiv: 1004.1296] [INSPIRE].

[99] M.F. Atiyah and I.M. Singer, The index of elliptic operators. 1, Annals Math. 87 (1968) 484 [INSPIRE].

[100] M.F. Atiyah and G.B. Segal, The Index of elliptic operators. 2, Annals Math. 87 (1968) 531 [INSPIRE].

[101] M.F. Atiyah and I.M. Singer, The Index of elliptic operators. 3, Annals Math. 87 (1968) 546 [INSPIRE].

[102] W. Zimmermann, Convergence of Bogolyubov's method of renormalization in momentum space, Commun. Math. Phys. 15 (1969) 208 [inSPIRE].

[103] J.H. Lowenstein, Differential vertex operations in Lagrangian field theory, Commun. Math. Phys. 24 (1971) 1 [INSPIRE].

[104] J.C. Collins, Normal products in dimensional regularization, Nucl. Phys. B 92 (1975) 477 [INSPIRE].

[105] P. Breitenlohner and D. Maison, Dimensional renormalization and the action principle, Commun. Math. Phys. 52 (1977) 11 [INSPIRE].

[106] N. Nakanishi, Covariant quantization of the electromagnetic field in the Landau gauge, Prog. Theor. Phys. 35 (1966) 1111 [INSPIRE].

[107] B. Lautrup, Canonical quantum electrodynamics in covariant gauges, Kong. Dan. Vid. Sel. Mat. Fys. Med. 35 (1967) 11 [INSPIRE].

[108] H. Lehmann, K. Symanzik and W. Zimmermann, On the formulation of quantized field theories, Nuovo Cim. 1 (1955) 205 [INSPIRE].

[109] C. Becchi, A. Rouet and R. Stora, The abelian Higgs-Kibble model. Unitarity of the $s$ operator, Phys. Lett. 52B (1974) 344 [INSPIRE].

[110] C. Becchi, A. Rouet and R. Stora, Renormalization of the abelian Higgs-Kibble model, Commun. Math. Phys. 42 (1975) 127 [InSPIRE]. 
[111] I.V. Tyutin, Gauge invariance in field theory and statistical physics in operator formalism, arXiv:0812.0580 [INSPIRE].

[112] M.Z. Iofa and I.V. Tyutin, Gauge invariance of spontaneously broken non-Abelian theories in the Bogolyubov-Parasyuk-Hepp-Zimmermann method, Theor. Math. Phys. 27 (1976) 316.

[113] C. Becchi, A. Rouet and R. Stora, Renormalization of gauge theories, Annals Phys. 98 (1976) 287 [INSPIRE].

[114] T. Kugo and I. Ojima, Manifestly covariant canonical formulation of Yang-Mills theories physical state subsidiary conditions and physical S-matrix unitarity, Phys. Lett. B $\mathbf{7 3}$ (1978) 459 [INSPIRE].

[115] T. Kugo and I. Ojima, Manifestly covariant canonical formulation of the Yang-Mills field theories. I. General formalism, Prog. Theor. Phys. 60 (1978) 1869 [InSPIRE].

[116] T. Kugo and I. Ojima, Local covariant operator formalism of nonabelian gauge theories and quark confinement problem, Prog. Theor. Phys. Suppl. 66 (1979) 1 [INSPIRE].

[117] G. 't Hooft and M.J.G. Veltman, Scalar one loop integrals, Nucl. Phys. B 153 (1979) 365 [INSPIRE].

[118] G. Passarino and M.J.G. Veltman, One loop corrections for $e^{+} e^{-}$annihilation into $\mu^{+} \mu^{-}$ in the Weinberg model, Nucl. Phys. B 160 (1979) 151 [INSPIRE].

[119] H.E. Logan, Radiative corrections to the Z Zb̄ vertex and constraints on extended Higgs sectors, Ph.D. thesis, University of California, Santa Cruz, U.S.A. (1999), hep-ph/9906332 [INSPIRE]. 